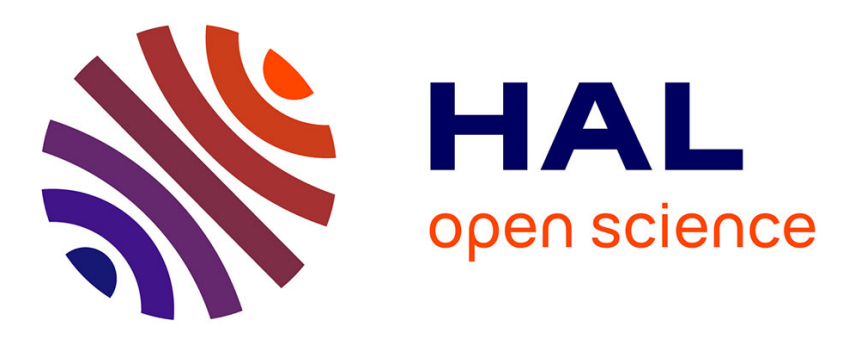

\title{
Inverse Problems for Time-Dependent Singular Heat Conductivities: Multi-Dimensional Case
}

Patricia Gaitan, Hiroshi Isozaki, Olivier Poisson, Samuli Siltanen, Janne Tamminen

\section{- To cite this version:}

Patricia Gaitan, Hiroshi Isozaki, Olivier Poisson, Samuli Siltanen, Janne Tamminen. Inverse Problems for Time-Dependent Singular Heat Conductivities: Multi-Dimensional Case. Communications in Partial Differential Equations, 2015, 40 (5), pp.837-877. 10.1080/03605302.2014.992533 . hal-01255481

\author{
HAL Id: hal-01255481 \\ https://hal.science/hal-01255481
}

Submitted on 13 Jan 2016

HAL is a multi-disciplinary open access archive for the deposit and dissemination of scientific research documents, whether they are published or not. The documents may come from teaching and research institutions in France or abroad, or from public or private research centers.
L'archive ouverte pluridisciplinaire HAL, est destinée au dépôt et à la diffusion de documents scientifiques de niveau recherche, publiés ou non, émanant des établissements d'enseignement et de recherche français ou étrangers, des laboratoires publics ou privés. 


\title{
INVERSE PROBLEMS FOR TIME-DEPENDENT SINGULAR HEAT CONDUCTIVITIES MULTI DIMENSIONAL CASE
}

\author{
P. GAITAN, H. ISOZAKI, O. POISSON, S. SILTANEN, AND J. TAMMINEN
}

\begin{abstract}
We consider an inverse boundary value problem for the heat equation $\partial_{t} u=\operatorname{div}\left(\gamma \nabla_{x} u\right)$ in $(0, T) \times \Omega, u=f$ on $(0, T) \times \partial \Omega,\left.u\right|_{t=0}=u_{0}$, in a bounded domain $\Omega \subset \mathbb{R}^{n}, n \geq 2$, where the heat conductivity $\gamma(t, x)$ is piecewise constant and the surface of discontinuity depends on time : $\gamma(t, x)=$ $k^{2}(x \in D(t)), \gamma(t, x)=1(x \in \Omega \backslash D(t))$. Fix a direction $\mathbf{e}^{*} \in \mathbb{S}^{n-1}$ arbitrarily. Assuming that $\partial D(t)$ is strictly convex for $0 \leq t \leq T$, we show that $k$ and $\sup \left\{e^{*} \cdot x ; x \in D(t)\right\}(0 \leq t \leq T)$, in particular $D(t)$ itself, are determined from the Dirichlet-to-Neumann map : $\left.f \rightarrow \partial_{\nu} u(t, x)\right|_{(0, T) \times \partial \Omega}$. The knowledge of the initial data $u_{0}$ is not used in the proof. If we know $\min _{0 \leq t \leq T}\left(\sup _{x \in D(t)} x\right.$. $\mathbf{e}^{*}$, we have the same conclusion from the local Dirichlet-to-Neumann map. The results have applications to nondestructive testing. Consider a physical body consisiting of homogeneous material with constant heat conductivity except for a moving inclusion with different conductivity. Then the location and shape of the inclusion can be monitored from temperature and heat flux measurements performed at the boundary of the body. Such a situation appears for example in blast furnaces used in ironmaking.
\end{abstract}

\section{INTRODUCTION}

1.1. Inverse heat conductivity problem. Let $\Omega$ be a bounded domain in $\mathbb{R}^{n}$, $n \geq 2$, with smooth boundary $\Gamma=\partial \Omega$, and consider the following initial boundary value problem

$$
\left\{\begin{aligned}
\partial_{t} u & =\operatorname{div}\left(\gamma \nabla_{x} u\right) \text { in }(0, T) \times \Omega, \\
u & =f \text { on }(0, T) \times \Gamma, \\
\left.u\right|_{t=0} & =u_{0} \text { on } \Omega
\end{aligned}\right.
$$

where $\gamma=\gamma(t, x)$ has the following properties : There exist a positive constant $k \neq 1$ and an open set $D(t) \subset \Omega$ with $C^{\infty}$ boundary $S(t)=\partial D(t)$ such that

$$
\begin{array}{r}
S(t) \cap \Gamma=\emptyset, \quad 0 \leq t \leq T, \\
\gamma(t, x)=\left\{\begin{array}{l}
1 \quad \text { if } \quad x \notin D(t), \\
k^{2} \quad \text { if } \quad x \in D(t),
\end{array}\right.
\end{array}
$$

and $[0, T] \ni t \rightarrow S(t)$ is also $C^{\infty}$. The following geometric condition is imposed:

(C-1) $\Omega$ and $D(t)$ are strictly convex for $0 \leq t \leq T$, i.e. all of the principal curvatures of the boundary are positive.

Date: February 6, 2013. 
Physically, the region $D(t)$ corresponds to some inclusion in the medium with heat conductivity different from that in the background domain $\Omega$. The problem we address in this paper is to determine $\gamma(t, x)$ by using the knowledge of the Dirichlet-to-Neumann map (D-N map) :

$$
\Lambda_{u_{0}, \gamma}: f \mapsto \partial_{\nu} u(t, x), \quad(t, x) \in(0, T) \times \Gamma,
$$

where $u=u_{u_{0}, \gamma}^{f}$ denotes the unique solution of (1.1), $\nu$ is the outer unit normal to $\Gamma$, and $\partial_{\nu}=\frac{\partial}{\partial \nu}=\nu \cdot \nabla_{x}$. In physical terms, $f=f(t, x)$ is the temperature distribution on the boundary and $\Lambda_{u_{0}, \gamma}(f)$ is the resulting heat flux through the boundary.

We shall consider a large parameter $\lambda>0$ and allow the initial data $u_{0}(x)$ to depend on $\lambda$, under the following condition:

(C-2) There exist constants $T_{0} \in[0, T), C>0$ such that $\left\|u_{0}\right\|_{L^{1}(\Omega)} \leq C e^{\lambda^{2} T_{0}}$.

The above inverse boundary value problem is related to nondestructive testing where one looks for anomalous materials inside a known material. One such example is monitoring a blast furnace used in ironmaking: the corroded thickness of the accreted refractory wall based on temperature and heat flux measurement on the accessible part of the furnace wall [19].

1.2. Main theorems. We fix a unit vector $\mathbf{e}^{*} \in \mathbb{S}^{n-1}$, and put

$$
h_{\mathrm{fW}}(t, x ; \lambda)=e^{\lambda^{2} t+\lambda x \cdot \mathbf{e}^{*}}, \quad h_{\mathrm{bW}}(t, x ; \lambda)=e^{-\lambda^{2} t+\lambda x \cdot \mathbf{e}^{*}},
$$

where $\lambda$ is a large parameter. Note that $h_{\mathrm{fw}}$ and $h_{\mathrm{bw}}$ solve the forward and the backward heat equation, respectively:

$$
\left(\partial_{t}-\Delta_{x}\right) h_{\mathrm{fw}}=0, \quad\left(\partial_{t}+\Delta_{x}\right) h_{\mathrm{bw}}=0 .
$$

Let $u(t, x ; \lambda)$ be the solution to $(1.1)$ with $f=h_{\mathrm{fw}}(t, x ; \lambda)$. We define, for $T_{1} \in$ $\left[T_{0}, T\right)$,

$$
I_{\Gamma}\left(T_{1}, T ; \lambda, \mu\right)=\int_{T_{1}}^{T} e^{\lambda \mu t}\left(\int_{\Gamma} h_{\mathrm{bw}}(t, x ; \lambda) \partial_{\nu}\left(u(t, x ; \lambda)-h_{\mathrm{fw}}(t, x ; \lambda)\right) d \Gamma\right) d t,
$$

where $d \Gamma$ is the surface element of $\Gamma$, and $\mu$ is a positive constant.

We put

$$
a(t)=\sup \left\{\mathbf{e}^{*} \cdot x ; x \in D(t)\right\}
$$

and $\dot{a}(t)=d a(t) / d t$. By the strict convexity (C-1), there exists a unique $y^{*}(t) \in S(t)$ such that

$$
a(t)=\mathbf{e}^{*} \cdot y^{*}(t) .
$$

and also, there is a unique $z^{*}(t) \in \Gamma$ such that

$$
\Gamma \cap\left\{y^{*}(t)+\rho \mathbf{e}^{*} ; \rho>0\right\}=\left\{z^{*}(t)\right\} .
$$


We put

$$
r(t)=\left|z^{*}(t)-y^{*}(t)\right| .
$$

Let $k_{i}(t), 1 \leq i \leq n-1$, be the principal curvatures of $S(t)$ at $y^{*}(t)$ and put

$$
K(t)=\prod_{i=1}^{n-1} k_{i}(t)
$$

Our first main theorem is as follows.

Theorem 1.1. Assume (C-1), (C-2). Then there exists a constant $\mu_{0}>0$ depending only on the geometry of $\Omega$ and $\|\dot{a}\|_{L^{\infty}\left(\left(T_{0}, T\right)\right)}$ such that if $\mu>\mu_{0}$ then $I_{\Gamma}\left(T_{0}, T ; \lambda, \mu\right)$ has the following asymptotic behavior as $\lambda \rightarrow \infty$ :

$$
I_{\Gamma}\left(T_{0}, T ; \lambda, \mu\right) \sim 2\left(\frac{\pi}{\lambda}\right)^{(n-1) / 2} \frac{1-k}{1+k} e^{\lambda \mu T+2 \lambda a(T)} \frac{e^{-r(T) \dot{a}(T)}}{\sqrt{K(T)}(\mu+2 \dot{a}(T))} .
$$

This theorem enables us to determine $k, a(t)$ and $S(t)$. Taking note that $u$ in Theorem 1.1 depends on $\mathbf{e}^{*} \in \mathbb{S}^{n-1}$, we denote $a(t)$ and $I_{\Gamma}\left(T_{0}, T ; \lambda, \mu\right)$ by, $a\left(t, \mathbf{e}^{*}\right)$ and $I_{\Gamma}\left(T_{0}, T ; \lambda, \mu, \mathbf{e}^{*}\right)$, respectively.

Corollary 1.2. Let $U$ be an open set in $\mathbb{S}^{n-1}$ and $\widetilde{U}=\{r \omega ; r>0, \omega \in U\}$. Then, from $\left\{I_{\Gamma}\left(T_{0}, T ; \lambda, \mu, \mathbf{e}^{*}\right) ; \mathbf{e}^{*} \in U, T_{0} \leq t \leq T\right\}$, one can reconstruct $k, a(t)$ and $S(t) \cap \widetilde{U}$ for $T_{0} \leq t \leq T$.

Remark 1.3. In Theorem 1.1 we have $\mu_{0} \geq 2\|\dot{a}\|_{L^{\infty}\left(\left(T_{0}, T\right)\right)}$.

We can also detect the inclusion by the local measurement. Take $a_{1}<a_{2}$, $\chi(s) \in C^{\infty}(\mathbb{R})$ such that $\chi(s)=1\left(s>a_{2}\right), \chi(s)>0\left(s>a_{1}\right), \chi(s)=0\left(s<a_{1}\right)$.

We put

$$
\begin{aligned}
& \widetilde{h_{\mathrm{fw}}}(t, x ; \lambda)=h_{\mathrm{fw}}(t, x ; \lambda) \chi\left(x \cdot \mathbf{e}^{*}\right), \\
& \widetilde{h_{\mathrm{bw}}}(t, x ; \lambda)=h_{\mathrm{bw}}(t, x ; \lambda) \chi\left(x \cdot \mathbf{e}^{*}\right),
\end{aligned}
$$

Put

$$
\Gamma_{1}=\Gamma \cap\left\{x \in \mathbb{R}^{n} ; x \cdot \mathbf{e}^{*}>a_{1}\right\},
$$

and remark that $\overline{\Gamma_{1}}=\operatorname{supp}\left(\widetilde{h_{\mathrm{fw}}}\right) \cap \Gamma=\operatorname{supp}\left(\widetilde{h_{\mathrm{bw}}}\right) \cap \Gamma$.

Theorem 1.4. Let $\widetilde{u}(t, x ; \lambda)$ be the solution to (1.1) with $f$ replaced by $\widetilde{h_{f w}}(t, x ; \lambda)$, and define the indicator function $\widetilde{I}_{\Gamma_{1}}\left(T_{0}, T ; \lambda, \mu\right)$ by

$$
\widetilde{I}_{\Gamma_{1}}\left(T_{0}, T ; \lambda, \mu\right)=\int_{T_{0}}^{T} e^{\lambda \mu t}\left(\int_{\Gamma_{1}} \widetilde{h_{b w}}(t, x ; \lambda) \partial_{\nu}\left(\widetilde{u}(t, x ; \lambda)-\widetilde{h_{f w}}(t, x ; \lambda)\right) d \Gamma\right) d t .
$$

Then we have

(1) If $a_{2}<a(T)$ then

$$
\widetilde{I}_{\Gamma_{1}}\left(T_{0}, T ; \lambda, \mu\right) \sim 2\left(\frac{\pi}{\lambda}\right)^{(n-1) / 2} \frac{1-k}{1+k} e^{\lambda \mu T+2 \lambda a(T)} \frac{e^{-r(T) \dot{a}(T)}}{\sqrt{K(T)}(\mu+2 \dot{a}(T))} .
$$


(2) If $a_{1}>a(T)$ then

$$
\left|\widetilde{I}_{\Gamma_{1}}\left(T_{0}, T ; \lambda, \mu\right)\right|=o\left(\lambda^{-N}\right) e^{\lambda \mu T+2 \lambda a_{2}}, \quad \forall N \in \mathbb{N} .
$$

Corollary 1.2 also holds with $I_{\Gamma}\left(T_{0}, T ; \lambda, \mu, \mathbf{e}^{*}\right)$ replaced by $\widetilde{I}_{\Gamma_{1}}\left(T_{0}, T ; \lambda, \mu\right)$.

1.3. Plan of the proof. In $\S 2$, we use J. L. Lions' theorem to prove the existence of solutions to the heat equation (1.1). There are two main steps in the proof of Theorem 1.1. The 1st one is the energy inequality to be prepared in $\S 3$. The 2 nd main step is the construction of approximate solution of the heat equation to be discussed in $\S 4$. As can be imagined easily, the first approximation of the solution to $(1.1)$ is

$$
e^{\lambda^{2} t}\left(b_{1}(t, x) e^{\lambda \mathbf{e}^{*} \cdot x}+b_{2}(t, x) e^{\lambda \Phi(t, x)}\right),
$$

where $b_{1}(t, x)$ and $b_{2}(t, x)$ are the characteristic functions of $\Omega \backslash D(t)$ and $D(t)$, respectively, and $\Phi(t, x)$ is a solution to the eikonal equation $\left|\nabla_{x} \Phi(t, x)\right|^{2}=1 / k^{2}$. Of course, this approximation is not sufficient, and we need to modify it in such a way that

$$
v(t, x)=e^{\lambda^{2} t} \sum_{j=1}^{4} b_{j}(t, x) e^{\lambda \Phi_{j}(t, x)},
$$

where $\Phi_{j}(t, x)$ is a suitable solution to the eikonal equation

$$
\left|\nabla_{x} \Phi_{j}(t, x)\right|^{2}=1, \quad 1 \leq j \leq 3, \quad\left|\nabla_{x} \Phi_{4}(t, x)\right|^{2}=\frac{1}{k^{2}},
$$

and the coefficients $b_{j}(t, x)$ are chosen to satisfy the compatibility conditions on $\partial D(t)$ to make $v(t, x)$ regular. We devote ourselves in $\S 4$ to construct solutions to the eikonal equation, and the transport equations. The approximate solution $v_{a p r}(t, x ; \lambda)$ will be constructed in $\S 5$. We also need to construct an approximate solution to the backward heat equation. In $\S 6$, we compare the exact solution $u(t, x)$ with $v_{a p r}(t, x ; \lambda)$ by using energy inequality. The choice of $\Phi_{j}(t, x)$ and the amplitude functions $b_{j}(t, x)$ must be done carefully in order to apply the energy estimates. The proof of Theorem 1.1 will then be completed in $\S 7$.

In the 1-dimensonal case, our results based on the localized data can be improved because of the simplicity of geometry. We shall summarize them in [7].

Throughtout the paper, we only deal with real-valued functions.

1.4. Related works. As in the elliptic equation, identification of inclusions is a typical issue in inverse problem for the parabolic equation, to which plenty of works have been devoted. However, most of them have treated the case in which the inclusion is independent of $t$. Main aims are the uniqueness and stability. Local the local uniqueness and stability was studied by Bellout [1], and global uniqueness was proved by Elayyan and Isakov [6] using the localized Neumann-to-Dirichlet (ND) map. Di Cristo and Vessella [3] obtained logarithmic stability estimates of the inclusion from the Dirichlet-to-Neumann map. Ikehata [12], and Ikehata-Kawashita [13] developed the probe method for the heat equation in the case that inclusions 
are time-independent. In [10], the case of time-independent inclusion was treated and the result for numerical computations was given. The idea is based on the complex spherical wave given by Ide-Isozaki-Nakata-Siltanen-Uhlmann [11] for the elliptic case. Other computational approaches to static inclusion detection include $[5,8]$.

The work of Daido, Kang and Nakamura [4] is close to the present paper. They studied the case of moving inclusions in dimension one: $D(t)=\left\{0<a_{0}(t)<x<\right.$ $\left.a_{1}(t)<1\right\}$ using the probe method, which is based on the explicit form of the heat kernel, and Runge's approximation theorem, and proved that $a_{1}(t)$ is obtained from the whole knowledge of Neumann-to-Dirichlet map. Their initial data is assumed to be $0: u_{0}(x)=0$, and the computation of $k$ was not done.

The present paper is different from all these works in the use of asymptotic heat flow and the energy inequalities. We remark that one-dimensional results similar to the present paper are published in [7].

As for the recent works on the inverse problem for the parabolic equation, see Bacchelli-Cristo-Sincich-Vessella [2] for the corrosion problem, and Vessella [20] and Kawakami-Tsuchiya [14] for the time-varying domain problem.

Computational approaches to a related moving boundary estimation problem in non-destructive testing have been published in [16, 17, 19]. The present results outline an algorithm potentially applicable to similar problems.

\section{Existence OF SOLUTiOns}

2.1. Abstract theorem. We begin with the well-posedness of (1.1), which is not obvious, since the coefficient is singular.

Let $\mathcal{H}$ and $\mathcal{H}_{1}$ be Hilbert spaces equipped with inner products $(),,(,)_{1}$ and norms $\|\cdot\|,\|\cdot\|_{1}$, respectively. Assume that $\mathcal{H}_{1}$ is a dense subspace of $\mathcal{H}$ and there exists a constant $C>0$ such that

$$
\|u\| \leq C\|u\|_{1}, \quad \forall u \in \mathcal{H}_{1} .
$$

Then we have the following inclusion relations

$$
\mathcal{H}_{1} \subset \mathcal{H} \subset \mathcal{H}_{1}^{*}
$$

For $t \in[0, T]$, let $a(t, \cdot, \cdot)$ be a quadratic form on $\mathcal{H}_{1} \times \mathcal{H}_{1}$ such that

$$
a(t, u, v)=\overline{a(t, v, u)}, \quad \forall u, v \in \mathcal{H}_{1}, \quad \forall t \in[0, T] .
$$

We also assume that there exist constants $\delta>0, C_{0}>0$ such that

$$
\begin{aligned}
& |a(t, u, v)| \leq C_{0}\|u\|_{1}\|v\|_{1}, \quad \forall u, v \in \mathcal{H}_{1}, \quad \forall t \in[0, T], \\
& a(t, u, u) \geq \delta\|u\|_{1}^{2}-C_{0}\|u\|^{2}, \quad \forall u \in \mathcal{H}_{1}, \quad \forall t \in[0, T] .
\end{aligned}
$$

The last assumption is :

$$
\text { For any } u, v \in \mathcal{H}_{1},[0, T] \ni t \rightarrow a(t, u, v) \text { is measurable. }
$$


These assumptions imply that there exists a unique self-adjoint operator $A(t)$ such that $D(A(t)) \subset \mathcal{H}_{1}$ and

$$
(A(t) u, v)=a(t, u, v), \quad \forall u \in D(A(t)), \quad \forall v \in \mathcal{H}_{1} .
$$

With this operator $A(t)$, we consider the following evolution equation on $\mathcal{H}$ :

$$
\left\{\begin{array}{l}
\partial_{t} u(t)+A(t) u(t)=f(t) \quad \text { in } \quad(0, T) \\
u(0)=u_{0} \in \mathcal{H}
\end{array}\right.
$$

We then have the following theorem due to J. L. Lions (see [15], [18]).

Theorem 2.1. Let $u_{0} \in \mathcal{H}$ and $f \in L^{2}\left((0, T) ; \mathcal{H}_{1}^{*}\right)$. Then there exists a unique $u(t)$ having the following properties.

(1) $u(t) \in C([0, T] ; \mathcal{H}) \cap L^{2}\left((0, T) ; \mathcal{H}_{1}\right)$.

(2) $u(t)$ is $\mathcal{H}_{1}^{*}$-valued absolutely continuous on $[0, T], \partial_{t} u(t) \in L^{2}\left((0, T) ; \mathcal{H}_{1}^{*}\right)$, and $u(t)$ satisfies (2.8).

(3) $u(t)$ satisfies the following (in) equalities :

$$
\begin{gathered}
\frac{1}{2}\|u(t)\|^{2}+\int_{0}^{t} a(s, u(s), u(s)) d s=\frac{1}{2}\left\|u_{0}\right\|^{2}+\int_{0}^{t}(f(s), u(s)) d s, \\
\|u(t)\|^{2}+\delta \int_{0}^{t}\|u(s)\|_{1}^{2} d s \leq\left\|u_{0}\right\|^{2}+\frac{1}{\delta} \int_{0}^{t}\|f(s)\|_{\mathcal{H}_{1}^{*}}^{2} d s .
\end{gathered}
$$

2.2. Heat equation. We take $\mathcal{H}=L^{2}(\Omega)$ and $\mathcal{H}_{1}=H_{0}^{1}(\Omega)$ which denotes the Sobolev space of order 1 with 0 trace on the boundary $\partial \Omega$. We put

$$
\Gamma=\partial \Omega, \quad S(t)=\partial D(t)
$$

and let $\nu_{\Gamma}=\left(\nu_{\Gamma}^{1}(x), \cdots, \nu_{\Gamma}^{n}(x)\right)$, and $\nu_{S(t)}=\left(\nu_{S(t)}^{1}, \cdots, \nu_{S(t)}^{n}\right)$ be the outer unit normals to $\Gamma$ and $S(t)$, respectively. We put

$$
\partial_{\nu_{\Gamma}}=\frac{\partial}{\partial \nu_{\Gamma}}=\nu_{\Gamma} \cdot \nabla_{x}, \quad \partial_{\nu_{S(t)}}=\frac{\partial}{\partial \nu_{S(t)}}=\nu_{S(t)} \cdot \nabla_{x},
$$

and omit the subscript $\Gamma$ or $S(t)$ for obvious cases. For a function $u(x)$, we define

$$
\begin{aligned}
& \left.u\right|_{S(t)} ^{( \pm)}(x)=\lim _{\epsilon \rightarrow 0, \epsilon>0} u\left(x \pm \epsilon \nu_{S(t)}\right), \\
& {[u]_{S(t)}(x)=\left.u\right|_{S(t)} ^{(+)}(x)-\left.u\right|_{S(t)} ^{(-)}(x) .}
\end{aligned}
$$

For $u, v \in H_{0}^{1}(\Omega)$, we put

$$
\begin{aligned}
a(t, u, v) & =\left(\gamma(t, x) \nabla_{x} u, \nabla_{x} v\right) \\
& =k^{2} \int_{D(t)} \nabla_{x} u(x) \cdot \overline{\nabla_{x} v(x)} d x+\int_{\Omega \backslash D(t)} \nabla_{x} u(x) \cdot \overline{\nabla_{x} v(x)} d x .
\end{aligned}
$$

Then the above assumptions $(2.3) \sim(2.6)$ are satisfied, and the associated $A(t)$ is given by

$$
D(A(t)) \ni u \Longleftrightarrow\left\{\begin{array}{l}
u \in H_{0}^{1}(\Omega) \cap H^{2}(D(t)) \cap H^{2}(\Omega \backslash D(t)), \\
\left.\partial_{\nu} u\right|_{S(t)} ^{(+)}=\left.k^{2} \partial_{\nu} u\right|_{S(t)} ^{(-)},
\end{array}\right.
$$




$$
A(t) u=\left\{\begin{array}{l}
-k^{2} \Delta u, \quad \text { on } \quad D(t), \\
-\Delta u, \quad \text { on } \quad \Omega \backslash D(t) .
\end{array}\right.
$$

In the following sections, we also use the notation $A(t)$ to denote the formal differential operator (2.17).

\section{ENERGy ESTIMATES}

We derive an energy inequality associated with the equation (1.1). We put

$$
\begin{gathered}
\mathcal{D}_{+}=\{(t, x) ; 0<t<T, x \in \Omega \backslash \overline{D(t)}\}, \\
\mathcal{D}_{-}=\{(t, x) ; 0<t<T, x \in D(t)\}, \\
E(v, H ; t)=\int_{\Omega}|v(t, x)| H(t, x) d x, \\
E_{\Gamma}(v, H ; t)=\int_{\Gamma}|v(t, x)| H(t, x) d \Gamma, \\
E_{S(t)}(v, H ; t)=\int_{S(t)}|v(t, x)| H(t, x) d S(t) .
\end{gathered}
$$

Theorem 3.1. Suppose $U(t, x)$ has the properties (1), (2) in Theorem 2.1 with $\mathcal{H}=L^{2}(\Omega), \mathcal{H}_{1}=H_{0}^{1}(\Omega)$. Assume also that $U$ satisfies the equation

$$
\partial_{t} U-\gamma(t, x) \Delta U=F \quad \text { in } \quad L^{2}\left(\mathcal{D}_{ \pm}\right) .
$$

Let $H(t, x) \in C^{2}\left(\overline{\mathcal{D}_{+}}\right) \cap C^{2}\left(\overline{\mathcal{D}_{-}}\right)$be such that

$$
\begin{gathered}
H(t, x) \geq 0 \quad \text { on } \quad[0, T] \times \Omega, \\
{[H]_{S(t)}=\left[\gamma \partial_{\nu} H\right]_{S(t)}=0, \quad 0 \leq t \leq T .}
\end{gathered}
$$

Assume that there exists a real constant $K$ such that

$$
\left.\partial_{t} H+\nabla_{x} \cdot\left(\gamma(t, x) \nabla_{x}\right) H\right) \leq-K H \quad \text { in } \quad(0, T) \times \Omega .
$$

Then we have the following inequality

$$
\begin{aligned}
& e^{K T} E(U, H ; T)+\int_{0}^{T} e^{K t} E_{\Gamma}\left(\partial_{\nu} U, H ; t\right) d t \\
\leq & E(U, H ; 0)+\int_{0}^{T} e^{K t} E(F, H ; t) d t+\int_{0}^{T} e^{K t} E_{S(t)}\left(\left[\gamma \partial_{\nu} U\right]_{S(t)}, H ; t\right) d t .
\end{aligned}
$$

Proof. Let $\chi_{\epsilon}(s)=s\left(\epsilon+s^{2}\right)^{-1 / 2}$ for $\epsilon>0$, and note the following properties :

$$
\begin{gathered}
s \chi_{\epsilon}(s) \rightarrow|s|, \quad \epsilon \rightarrow 0, \\
\chi_{\epsilon}^{\prime}(s)>0, \quad\left|s \chi_{\epsilon}^{\prime}(s)\right| \leq 1 / 2, \quad s \chi_{\epsilon}^{\prime}(s) \rightarrow 0, \quad \epsilon \rightarrow 0 .
\end{gathered}
$$

In fact, (3.12) follows from $\chi_{\epsilon}^{\prime}(s)=\epsilon\left(\epsilon+s^{2}\right)^{-3 / 2}$, and the fact that, putting $s=\sqrt{\epsilon} y$, $\left|s \chi_{\epsilon}^{\prime}(s)\right|=\left|y\left(1+y^{2}\right)^{-3 / 2}\right| \leq 1 / 2$. 
Note that the traces of the normal derivative of $\left.U\right|_{\mathcal{D} \text {. }}(t, \cdot)$ on the interface $S(t)$ is well-defined, for all positive $t$. By Green's formula, we have if $[f]_{S(t)}=[g]_{S(t)}=0$,

$$
\begin{aligned}
\int_{\Omega}((\gamma \Delta f) g-f(\gamma \Delta g)) d x= & \int_{\Gamma}\left(\left(\partial_{\nu} f\right) g-f\left(\partial_{\nu} g\right)\right) d \Gamma \\
& -\int_{S(t)}\left(\left[\gamma \partial_{\nu} f\right]_{S(t)} g-f\left[\gamma \partial_{\nu} g\right]_{S(t)}\right) d S(t) .
\end{aligned}
$$

We take $f=U \chi_{\epsilon}(U)$ and $g=H$. Thanks to $\left.U(t)\right|_{\Gamma}=0,[U]_{S(t)}=0$, and to (3.8), we have

$$
\begin{gathered}
\left.f\right|_{\Gamma}=\left.\partial_{\nu} f\right|_{\Gamma}=0, \quad\left[\gamma \partial_{\nu} g\right]_{S(t)}=0 \\
{\left[\gamma \partial_{\nu} f\right]_{S(t)}=\left.\left[\gamma \partial_{\nu} U\right]_{S(t)}\left(\chi_{\epsilon}(U)+U \chi_{\epsilon}^{\prime}(U)\right)\right|_{S(t)} .}
\end{gathered}
$$

Therefore, we have by (3.13)

$$
\int_{\Omega}((\gamma \Delta f) g-f(\gamma \Delta g)) d x=-\int_{S(t)}\left[\gamma \partial_{\nu} f\right]_{S(t)} g d S(t) .
$$

By integration by parts, we have

$$
\begin{aligned}
\int_{\Omega}\left(\gamma \Delta \chi_{\epsilon}(U)\right) U H d x= & -\int_{S(t)} \chi_{\epsilon}^{\prime}(U)\left[\gamma \partial_{\nu} U\right]_{S(t)} U H d S(t) \\
& -\int_{\Omega} \gamma \chi_{\epsilon}^{\prime}(U)\left(|\nabla U|^{2} H+U \nabla U \cdot \nabla H\right) d x
\end{aligned}
$$

Plugging this with (3.14), we obtain

$$
\begin{aligned}
& \int_{\Omega}(\gamma \Delta U) \chi_{\epsilon}(U) H d x-\int_{\Omega} U \chi_{\epsilon}(U)(\gamma \Delta H) d x \\
& =-\int_{S(t)}\left[\gamma \partial_{\nu} U\right]_{S(t)} \chi_{\epsilon}(U) H d S(t)-\int_{\Omega} \gamma|\nabla U|^{2} \chi_{\epsilon}^{\prime}(U) H d x \\
& \quad+\int_{\Omega} U \chi_{\epsilon}^{\prime}(U) \gamma \nabla U \cdot \nabla H d x .
\end{aligned}
$$

We put

$$
E_{\epsilon}(t)=\int_{\Omega} U(t, x) \chi_{\epsilon}(U(x, t)) H(t, x) d x .
$$

Then we have by using (3.6)

$$
\begin{aligned}
\partial_{t} E_{\epsilon}(t)= & \int_{\Omega}(\gamma \Delta U) \chi_{\epsilon}(U) H d x-\int_{\Omega} U \chi_{\epsilon}(U)(\gamma \Delta H) d x \\
& +\int_{\Omega} F \chi_{\epsilon}(U) H d x+\int_{\Omega} U \chi_{\epsilon}(U)\left(\partial_{t} H+\gamma \Delta H\right) d x \\
& +\int_{\Omega} U \chi_{\epsilon}^{\prime}(U) \partial_{t} U H d x
\end{aligned}
$$


Plugging this with (3.15), we have

$$
\begin{aligned}
\partial_{t} E_{\epsilon}(t)= & -\int_{S(t)}\left[\gamma \partial_{\nu} U\right]_{S(t)} \chi_{\epsilon}(U) H d S(t)-\int_{\Omega} \gamma|\nabla U|^{2} \chi_{\epsilon}^{\prime}(U) H d x \\
& +\int_{\Omega} U \chi_{\epsilon}^{\prime}(U) \gamma \nabla U \cdot \nabla H d x+\int_{\Omega} F \chi_{\epsilon}(U) H d x \\
& +\int_{\Omega} U \chi_{\epsilon}(U)\left(\partial_{t} H+\gamma \Delta H\right) d x+\int_{\Omega} U \chi_{\epsilon}^{\prime}(U) \partial_{t} U H d x .
\end{aligned}
$$

By (3.12), the integrals containing the term $U \chi_{\epsilon}^{\prime}(U)$ vanish as $\epsilon \rightarrow 0$. Using (3.9), we then have

$$
\begin{aligned}
& \partial_{t} E_{\epsilon}(t)+K E_{\epsilon}(t)+\int_{\Omega} \gamma|\nabla U|^{2} \chi_{\epsilon}^{\prime}(U) H d x \\
\leq & -\int_{S(t)}\left[\gamma \partial_{\nu} U\right]_{S(t)} \chi_{\epsilon}(U) H d S(t)+\int_{\Omega} F \chi_{\epsilon}(U) H d x+o(1) .
\end{aligned}
$$

We multiply this inequality by $e^{K t}$ and integrate on the time interval $[0, T]$ to obtain

$$
\begin{aligned}
& e^{K T} E_{\epsilon}(T)+\int_{0}^{T} e^{K t} d t \int_{\Omega} \gamma|\nabla U|^{2} \chi_{\epsilon}^{\prime}(U) H d x \\
\leq & E_{\epsilon}(0)-\int_{0}^{T} e^{K t} d t \int_{S(t)}\left[\gamma \partial_{\nu} U\right]_{S(t)} \chi_{\epsilon}(U) H d S(t) \\
& +\int_{0}^{T} e^{K t} d t \int_{\Omega} F \chi_{\epsilon}(U) H d x+o(1) .
\end{aligned}
$$

Here we make use of the following lemma.

Lemma 3.2. For a sufficiently small $\delta>0$, let $\Omega_{\delta}=\{x \in \Omega$; $\operatorname{dist}(x, \Gamma)<\delta\}$. Suppose that $U \in H^{2}\left(\Omega_{\delta}\right)$ satisfies $U=0$ on $\Gamma$. Then for any nonnegative function $a(x) \in C\left(\overline{\Omega_{\delta}}\right)$, we have

$$
\liminf _{\epsilon \rightarrow 0} \int_{\Omega_{\delta}} a(x)|\nabla U(x)|^{2} \frac{\epsilon}{\left(\epsilon+U(x)^{2}\right)^{3 / 2}} d x \geq \int_{\Gamma} a(x)|\nabla U(x)| d \Gamma .
$$

Granting this lemma, we continue the proof of Theorem 3.1. Taking $a=H$ in Lemma 3.2 and using $\chi_{\epsilon}^{\prime}(U) \geq 0$ by (3.12), we have

$$
\int_{\Gamma}|\nabla U| H d \Gamma \leq \liminf _{\epsilon \rightarrow 0} \int_{\Omega} \gamma|\nabla U|^{2} \chi_{\epsilon}^{\prime}(U) H d x
$$

Noting this and (3.11), taking the inferior limit in (3.16), we conclude Theorem 3.1. Here we use the fact that $|\nabla U|=\left|\partial_{\nu} U\right|$ on $\Gamma$, since $U=0$ on $\Gamma$.

Proof of Lemma 3.2. Let $V$ be a small open set in $\Gamma$ on which $\nabla U \neq 0$. Without loss of generality, we assume that $\partial U / \partial x_{n} \neq 0$ on $V$, and take local coordinates $z_{i}=x_{i}(1 \leq i \leq n-1)$. Letting $z_{n}=U(x)$, we then have $d x=d \Gamma d z_{n} /|\nabla U|$. Take $\chi(x) \in C_{0}^{\infty}\left(\mathbb{R}^{n}\right)$ with small support such that $0 \leq \chi \leq 1$, and $\chi=1$ on $V$. Then we have

$$
\int_{\Omega_{\delta}} a(x)|\nabla U(x)|^{2} \frac{\epsilon}{\left(\epsilon+U(x)^{2}\right)^{3 / 2}} d x \geq \int_{\Omega^{\prime}} \chi(x) a(x)|\nabla U| \frac{\epsilon}{\left(\epsilon+z_{n}^{2}\right)^{3 / 2}} d \Gamma d z_{n},
$$


where $\Omega^{\prime}=\Gamma \times\left(0, \delta_{0}\right)$ with sufficiently small $\delta_{0}>0$. Putting $z_{n}=\sqrt{\epsilon} \rho$, the right-hand side is rewritten as

$$
\int_{\Gamma} d \Gamma \int_{0}^{\delta_{0} / \sqrt{\epsilon}} \chi(x) a(x)|\nabla U(x)| \frac{1}{\left(1+\rho^{2}\right)^{3 / 2}} d \rho .
$$

As $\epsilon \rightarrow 0$, this converges to

$$
\int_{\Gamma} \chi(x) a(x)|\nabla U(x)| d \Gamma
$$

since $\int_{0}^{\infty}\left(1+\rho^{2}\right)^{-3 / 2} d \rho=1$. Therefore, we have proven

$$
\liminf _{\epsilon \rightarrow 0} \int_{\Omega_{\delta}} a(x)|\nabla U(x)|^{2} \frac{\epsilon}{\left(\epsilon+U(x)^{2}\right)^{3 / 2}} d x \geq \int_{V} a(x)|\nabla U(x)| d \Gamma .
$$

Enlarging $V$, we obtain the lemma.

Let $u(t, x ; \lambda)$ be the solution to $(1.1)$ with $f=h_{\mathrm{fW}}(t, x ; \lambda)$. A first application of the energy estimate is the following lemma 3.3 , which gives a bound of $u\left(T_{1}, \cdot ; \lambda\right)$, $T_{1} \in\left[T_{0}, T\right]$, in the space $L^{1}(\Omega)$.

Lemma 3.3. Let $T_{1} \in\left[T_{0}, T\right]$. Then there exist constants $C>0, \lambda_{0}>0$ such that for all $\lambda \geq \lambda_{0}$

$$
\left\|u\left(T_{1}, \cdot ; \lambda\right)\right\|_{L^{1}(\Omega)}+\int_{0}^{T_{1}}\left\|\partial_{\nu}\left(u(t, \cdot ; \lambda)-h_{f w}(t, \cdot ; \lambda)\right)\right\|_{L^{1}(\Gamma)} d t \leq C e^{\lambda^{2} T_{1}+\lambda \rho_{0}},
$$

where $\rho_{0}$ is the diameter of $\Omega$.

Proof. If $T_{1}=T_{0}=0$ the estimates is obvious with $\rho_{0}=0$. So we assume that $T_{1}>0$. Let $U=u-h_{\mathrm{fw}}(\cdot ; \lambda)$. Then, $U=0$ on $\Gamma$,

$$
\left|\partial_{t} U-\gamma \Delta_{x} U\right| \leq \lambda^{2}\left|1-k^{2}\right| e^{\lambda^{2} t+\lambda a(t)} \quad \text { in } \quad L^{2}\left(\mathcal{D}_{ \pm}\right),
$$

and, for $0<t \leq T$,

$$
\left|\left[\gamma \partial_{\nu} U\right]_{S(t)}\right| \leq \lambda|1-k| e^{\lambda^{2} t+\lambda a(t)} .
$$

We apply Theorem 3.1 in the cylinder $\left(0, T_{1}\right) \times \Omega$ with $H=1$ and $K=0$. Then we have

$$
\begin{array}{r}
\left\|U\left(T_{1}, \cdot ; \lambda\right)\right\|_{L^{1}(\Omega)}+\int_{0}^{T_{1}} \int_{\Gamma}\left|\partial_{\nu} U(t, x ; \lambda)\right| d \Gamma d t \\
\leq\left\|u_{0}-e^{\lambda x \cdot \mathbf{e}^{*}}\right\|_{L^{1}(\Omega)}+C\left(\lambda^{2}+\lambda\right) \int_{0}^{T_{1}} e^{\lambda^{2} t+\lambda a(t)} d t .
\end{array}
$$

Thanks to (C-2) we have

$$
\left\|u_{0}-e^{\lambda x \cdot \mathbf{e}^{*}}\right\|_{L^{1}(\Omega)} \leq C e^{\lambda^{2} T_{0}}+C e^{\lambda \sup \left\{x \cdot \mathbf{e}^{*} ; x \in \Omega\right\}} .
$$

Thanks to (6.6) we have

$$
\left(\lambda^{2}+\lambda\right) \int_{0}^{T_{1}} e^{\lambda^{2} t+\lambda a(t)} d t \leq C e^{\lambda^{2} T_{1}+\lambda \sup \left\{x \cdot \mathbf{e}^{*} ; x \in \Omega\right\}} .
$$

Hence we have, for all $\lambda>\lambda_{0}$,

$$
\left\|U\left(T_{1}, \cdot ; \lambda\right)\right\|_{L^{1}(\Omega)}+\int_{0}^{T_{1}} \int_{\Gamma}\left|\partial_{\nu} U(t, x ; \lambda)\right| d \Gamma d t \leq C e^{\lambda^{2} T_{1}+\lambda \max \left(0, \sup \left\{x \cdot \mathbf{e}^{*} ; x \in \Omega\right\}\right)} .
$$


Observing that

$$
\left\|u\left(T_{1}, \cdot ; \lambda\right)\right\|_{L^{1}(\Omega)} \leq\left\|U\left(T_{1}, \cdot ; \lambda\right)\right\|_{L^{1}(\Omega)}+C e^{\lambda^{2} T_{1}+\lambda \sup \left\{x \cdot \mathbf{e}^{*} ; x \in \Omega\right\}},
$$

the conclusion follows.

This lemma enables us to regard $t=T_{1}$ as the initial time.

\section{Eikonal EQUations}

4.1. Geometric setting. To avoid the complexity of notation, we consider the case that $\mathbf{e}^{*}=\mathbf{e}_{\mathbf{n}}=(0, \cdots, 0,1)$. We denote points $x \in \mathbb{R}^{n}$ as $x=\left(x^{\prime}, x_{n}\right)$, $x^{\prime}=\left(x_{1}, \ldots, x_{n-1}\right)$. Since $\Omega$ is convex, for any $x \in \Omega$ there exists a unique $z(x)=$ $\left(z^{\prime}(x), z_{n}(x)\right) \in \Gamma$ such that

$$
z^{\prime}(x)=x^{\prime}, \quad z_{n}(x)>x_{n}, \quad \nu_{\Gamma}(z(x)) \cdot \mathbf{e}_{\mathbf{n}}>0 .
$$

Recall that $\nu_{\Gamma}$ and $\nu_{S(t)}$ are the outer unit normals to $\Gamma$ and $S(t)$.

As in $\S 1, y^{*}(t) \in S(t)$ is the point such that

$$
a(t)=\sup _{y \in S(t)} y \cdot \mathbf{e}^{*}=y^{*}(t) \cdot \mathbf{e}^{*} .
$$

Note that $\nu_{S(t)}\left(y^{*}(t)\right)=\mathbf{e}_{\mathbf{n}}$, since $\mathbf{e}^{*}=\mathbf{e}_{\mathbf{n}}$. We set

$$
z^{*}(t)=z\left(y^{*}(t)\right), \quad r(t)=\left|z^{*}(t)-y^{*}(t)\right|,
$$

which agree with the definitions in $\S 1$.

In a small neighborhood of $y^{*}(t), S(t)$ is written as

$$
x \in S(t) \Longleftrightarrow x_{n}=\varphi\left(t, x^{\prime}\right),
$$

with a smooth function $\varphi\left(t, x^{\prime}\right)$. Since $\varphi\left(t, x^{\prime}\right)$ attains a local maximum at $y^{*}(t)^{\prime}$, and $S(t)$ is strictly convex, we have

$$
\varphi\left(t, x^{\prime}\right)=\varphi\left(t, y^{*}(t)^{\prime}\right)-\frac{1}{2}\left\langle A(t)\left(x^{\prime}-y^{*}(t)^{\prime}\right),\left(x^{\prime}-y^{*}(t)^{\prime}\right)\right\rangle+O\left(\left|x^{\prime}-y^{*}(t)^{\prime}\right|^{3}\right),
$$

where $A(t)$ is a positive definite matrix, and $\langle$,$\rangle denotes the inner product of \mathbb{R}^{n-1}$. For small $\epsilon>0$, we put

$$
\begin{gathered}
D_{\epsilon,+}(t)=\left\{\left(x^{\prime}, x_{n}\right) \in \Omega ;\left|x^{\prime}-y^{*}(t)^{\prime}\right|<\epsilon, \varphi\left(t, x^{\prime}\right)<x_{n}\right\}, \\
D_{\epsilon,-}(t)=\left\{\left(x^{\prime}, x_{n}\right) \in \Omega ;\left|x^{\prime}-y^{*}(t)^{\prime}\right|<\epsilon, a(t)-\epsilon^{2}<x_{n}<\varphi\left(t, x^{\prime}\right)\right\} .
\end{gathered}
$$

We also put

$$
\begin{gathered}
S_{\epsilon}(t)=S(t) \cap\left\{\left|x^{\prime}-y^{*}(t)^{\prime}\right|<\epsilon, a(t)-\epsilon^{2}<x_{n}\right\}, \\
\Gamma_{\epsilon}(t)=\Gamma \cap\left\{\left|x^{\prime}-y^{*}(t)^{\prime}\right|<\epsilon, \varphi\left(t, x^{\prime}\right)<x_{n}\right\} .
\end{gathered}
$$

We are going to construct three solutions of the eikonal equation

$$
\left|\nabla_{x} \Phi(t, x)\right|^{2}=1,
$$

in the region $D_{\epsilon,+}(t)$ (or in its subregion), and one solution to the equation

$$
\left|\nabla_{x} \Phi(t, x)\right|^{2}=\frac{1}{k^{2}}
$$


in the region $D_{\epsilon,-}(t)$ satisfying appropriate boundary conditions. In the following, $\epsilon$ is chosen locally independently of $t$, hence globally independently of $t \in[0, T]$.

4.2. Eikonal equations in $D_{\epsilon,+}(t)$. We put $\partial_{j}=\partial / \partial x_{j}$ and $\nabla^{\prime}=\left(\partial_{1}, \cdots, \partial_{n-1}\right)$.

Lemma 4.1. There exist two solutions $\Phi_{1}(t, x), \Phi_{2}(t, x)$ of $(4.9)$ in $D_{\epsilon,+}(t)$ such that

$$
\begin{gathered}
\Phi_{1}(t, x)=\Phi_{2}(t, x)=\varphi\left(t, x^{\prime}\right), \quad \text { on } \quad S_{\epsilon}(t), \\
\frac{\partial}{\partial x_{n}} \Phi_{1}(t, x)>0, \quad \frac{\partial}{\partial x_{n}} \Phi_{2}(t, x)<0, \quad \text { on } \quad S_{\epsilon}(t) .
\end{gathered}
$$

They are written as

$$
\begin{gathered}
\Phi_{1}(t, x)=x_{n}, \quad x \in D_{\epsilon,+}(t) \\
\Phi_{2}(t, x)=-|x-y|+\varphi\left(t, y^{\prime}\right), \quad x \in D_{\epsilon,+}(t),
\end{gathered}
$$

where $y=\left(y^{\prime}, \varphi\left(t, y^{\prime}\right)\right) \in S_{\epsilon}(t)$ satisfies the equation

$$
\begin{gathered}
p\left(t, y^{\prime}\right)=\frac{y-x}{|y-x|}, \\
p_{j}=\frac{2 \partial_{j} \varphi}{\left|\nabla^{\prime} \varphi\right|^{2}+1}, \quad 1 \leq j \leq n-1, \quad p_{n}=\frac{\left|\nabla^{\prime} \varphi\right|^{2}-1}{\left|\nabla^{\prime} \varphi\right|^{2}+1} .
\end{gathered}
$$

Proof. Let us recall the standard method of solving the eikonal equation. Let $\left(y_{1}, \cdots, y_{n-1}\right)=\left(x_{1}, \cdots, x_{n-1}\right)$ be the local coordinates on $S_{\epsilon}(t)$ and put $y_{n}=$ $\varphi\left(t, y^{\prime}\right)$. Then the characteristic curves associated with (4.9) are straight lines given by

$$
x=2 s p+y, \quad y \in S_{\epsilon}(t),
$$

where $s$ is the parameter along the curve, and $p=p(y)$ satisfies, as a 1 form on $S_{\epsilon}(t)$,

$$
d \varphi=\sum_{j=1}^{n} p_{j} d x_{j} \quad \text { and } \quad \sum_{j=1}^{n} p_{j}^{2}=1, \quad \text { on } \quad S_{\epsilon}(t) .
$$

We then have as a 1 -form on $\mathbb{R}^{n}$

$$
\begin{aligned}
d \Phi & =\sum_{j=1}^{n} p_{j} d x_{j}=\sum_{j=1}^{n} p_{j}\left(2 s d p_{j}+2 p_{j} d s+d y_{j}\right) \\
& =s d|p|^{2}+2|p|^{2} d s+\sum_{j=1}^{n} p_{j} d y_{j}=2 d s+d \varphi
\end{aligned}
$$

which implies

$$
\Phi=2 s+\varphi .
$$

Note that $\nabla_{x} \Phi=p$ and $|p|=1$. 
We rewrite the first equation of (4.18) as

$$
d \varphi=\sum_{j=1}^{n-1} \partial_{j} \varphi d y_{j}=\sum_{j=1}^{n-1}\left(p_{j}+p_{n} \partial_{j} \varphi\right) d y_{j} .
$$

Therefore $p_{j}=\partial_{j} \varphi\left(1-p_{n}\right)$, and we have using $|p|=1$

$$
\left(\left|\nabla^{\prime} \varphi\right|^{2}+1\right) p_{n}^{2}-2\left|\nabla^{\prime} \varphi\right|^{2} p_{n}+\left|\nabla^{\prime} \varphi\right|^{2}-1=0,
$$

which determines $p_{n}$ i.e.

$$
p_{n}=1, \quad p_{n}=\frac{\left|\nabla^{\prime} \varphi\right|^{2}-1}{\left|\nabla^{\prime} \varphi\right|^{2}+1} .
$$

Adopting the former, we get $p=(0, \cdots, 0,1)$, and (4.13). Adopting the latter, we have (4.16). Since $\partial_{n} \Phi_{2}=p_{n}=-1+O\left(\left|y^{\prime}-y^{*}(t)^{\prime}\right|^{2}\right)$, the condition (4.12) is satisfied. Since $p$ is downward, $s<0$. Therefore by (4.17), we have

$$
2 s=-|x-y| /|p|=-|x-y|,
$$

which proves (4.14). This and (4.17) imply (4.15).

Lemma 4.2. There exists $\Phi_{3}(t, x)$ satisfying (4.9) near $\Gamma_{\epsilon}(t)$ and

$$
\begin{gathered}
\Phi_{3}(t, x)=\Phi_{2}(t, x), \quad \text { on } \quad \Gamma_{\epsilon}(t), \\
\partial_{\nu} \Phi_{3}(t, x)>0, \quad \text { on } \quad \Gamma_{\epsilon}(t) . \\
\partial_{\nu} \Phi_{3}(t, x)=-\partial_{\nu} \Phi_{2}(t, x), \quad \text { on } \Gamma_{\epsilon}(t) .
\end{gathered}
$$

Proof. As above the characteristic curves are straight lines defined by

$$
x=2 \bar{s} \bar{p}+z, \quad z \in \Gamma_{\epsilon},
$$

$\bar{p}=\bar{p}(z)$ such that $|\bar{p}|=1$, and $\Phi(x)=2 \bar{s}+\Phi_{2}(t, z)$. We construct $\bar{p}_{j}$ on $\Gamma_{\epsilon}(t)$ such that

$$
d \Phi_{2}=\sum_{j=1}^{n} \bar{p}_{j} d x_{j} \quad \text { and } \quad \sum_{j=1}^{n}\left|\bar{p}_{j}\right|^{2}=1, \quad \text { on } \quad \Gamma_{\epsilon}(t) .
$$

We assume that $\Gamma_{\epsilon}(t)$ is written as $z_{n}=\psi\left(t, z^{\prime}\right)$, and take $\left(z_{1}, \cdots, z_{n-1}\right)=$ $\left(x_{1}, \cdots, x_{n-1}\right)$ as local coordinates. Then, as above, we get

$$
\bar{p}_{j}=\partial_{j} \Phi_{2}+\left(\partial_{n} \Phi_{2}-\bar{p}_{n}\right) \partial_{j} \psi, \quad 1 \leq j \leq n-1 .
$$

Using $|\bar{p}|^{2}=1$, we have

$$
\begin{aligned}
& \left(\left|\nabla^{\prime} \psi\right|^{2}+1\right) \bar{p}_{n}^{2}-2\left(\nabla^{\prime} \psi \cdot \nabla^{\prime} \Phi_{2}+\left|\nabla^{\prime} \psi\right|^{2} \partial_{n} \Phi_{2}\right) \bar{p}_{n} \\
& +\left|\nabla^{\prime} \psi\right|^{2}\left(\partial_{n} \Phi_{2}\right)^{2}+2\left(\nabla^{\prime} \psi \cdot \nabla^{\prime} \Phi_{2}\right) \partial_{n} \Phi_{2}-\left(\partial_{n} \Phi_{2}\right)^{2}=0 .
\end{aligned}
$$

Solving this equation, we get

$$
\bar{p}_{n}=\partial_{n} \Phi_{2}, \quad \bar{p}_{n}=\frac{2 \nabla^{\prime} \psi \cdot \nabla^{\prime} \Phi_{2}+\left(\left|\nabla^{\prime} \psi\right|^{2}-1\right) \partial_{n} \Phi_{2}}{\left|\nabla^{\prime} \psi\right|^{2}+1} .
$$

Since $\nabla \psi=O\left(\left|z^{\prime}-z^{*}(t)^{\prime}\right|\right)$, the latter solution is close to $-\partial_{n} \Phi_{2}$. Recall that along the segment with end points $y^{*}(t)$ and $z^{*}(t), \Phi_{2}(t, x)=2 y_{n}^{*}(t)-x_{n}$ and $\nabla \Phi_{2}(t, x)=(0, \cdots, 0,-1)$. In particular, $\partial_{n} \Phi_{2}=-1$ at $x=z^{*}(t)$. Therefore, 
$\partial_{n} \Phi_{2}$ is close to -1 on $\Gamma_{\epsilon}(t)$. We thus adopt the latter solution to get $\bar{p}_{n}>0$, $\bar{s} \leq 0$. This proves $(4.21)$. To prove $(4.22)$, we have only to note that $(-\nabla \psi, 1)$ is an outward normal to $\Gamma_{\epsilon}(t)$, and to do a direct computation.

\subsection{Eikonal equations in $D_{\epsilon,-}(t)$.}

Lemma 4.3. There exist $\Phi_{4}(t, x)$ satisfying (4.10) in $D_{\epsilon,-}(t)$, and

$$
\Phi_{4}(t, x)=\varphi\left(t, x^{\prime}\right) \quad \text { and } \quad \partial_{\nu} \Phi_{4}(t, x)>0, \quad \text { on } \quad S_{\epsilon}(t) .
$$

Moreover, letting $p_{j}=\partial_{j} \Phi_{4}$, we have

$$
\begin{gathered}
p_{j}=2 \partial_{j} \varphi \frac{1-\frac{1}{k} \sqrt{1+\left(1-k^{2}\right)\left|\nabla^{\prime} \varphi\right|^{2}}}{\left|\nabla^{\prime} \varphi\right|^{2}+1}, \quad 1 \leq j \leq n-1, \\
p_{n}=\frac{\left|\nabla^{\prime} \varphi\right|^{2}+\frac{1}{k} \sqrt{1+\left(1-k^{2}\right)\left|\nabla^{\prime} \varphi\right|^{2}}}{\left|\nabla^{\prime} \varphi\right|^{2}+1} .
\end{gathered}
$$

Proof. We construct $p$ such that

$$
d \varphi=\sum_{j=1}^{n} p_{j} d x_{j}, \quad \sum_{j=1}^{n} p_{j}^{2}=\frac{1}{k^{2}}, \quad \text { on } \quad S_{\epsilon}(t) .
$$

As in the proof of Lemma 4.1, $p_{j}=2 \partial_{j} \varphi\left(1-p_{n}\right)$, hence $p_{n}$ satisfies

$$
\left(\left|\nabla^{\prime} \varphi\right|^{2}+1\right) p_{n}^{2}-2\left|\nabla^{\prime} \varphi\right|^{2} p_{n}+\left|\nabla^{\prime} \varphi\right|^{2}-1 / k^{2}=0 .
$$

Since $\nabla^{\prime} \varphi=O\left(\left|y^{\prime}-y^{*}(t)^{\prime}\right|\right)$, the solution is close to that of $p_{n}^{2}=1 / k^{2}$. Therefore, we can find a solution $p_{n}>0$. Then the lemma can be shown in the same way as Lemma 4.1.

4.4. Transport equations. Let us note the following identity:

$$
\begin{aligned}
e^{-\lambda^{2} t-\lambda \Phi}\left(\partial_{t}-\Delta_{x}\right)\left(a e^{\lambda^{2} t+\lambda \Phi}\right)= & \lambda^{2}\left(1-\left|\nabla_{x} \Phi\right|^{2}\right) a \\
& -\lambda\left(2 \nabla_{x} \Phi \cdot \nabla_{x} a+\left(\Delta_{x} \Phi-\partial_{t} \Phi\right) a\right) \\
& +\partial_{t} a-\Delta_{x} a .
\end{aligned}
$$

Letting $\Phi$ to be a solution to the eikonal equation, and $a=\sum_{i=0}^{N} \lambda^{-i} a_{i}$, we can rewrite the right-hand side as

$$
\begin{aligned}
& -\lambda\left(2 \nabla_{x} \Phi \cdot \nabla a_{0}+\left(\Delta_{x} \Phi-\partial_{t} \Phi\right) a_{0}\right) \\
& -\sum_{i=1}^{N} \lambda^{1-i}\left(\left(2 \nabla_{x} \Phi \cdot \nabla_{x}+\left(\Delta_{x} \Phi-\partial_{t} \Phi\right)\right) a_{i}-\left(\partial_{t}-\Delta_{x}\right) a_{i-1}\right) \\
& +\lambda^{-N}\left(\partial_{t}-\Delta_{x}\right) a_{N} .
\end{aligned}
$$


The 1st and 2nd terms vanish if we put, by integrating along the characteristic curve,

$$
\begin{aligned}
a_{0}= & a_{00} \exp \left(\int_{0}^{s}\left(\partial_{t} \Phi-\Delta_{x} \Phi\right) d \tau\right) \\
a_{i}= & a_{i 0} \exp \left(\int_{0}^{s}\left(\partial_{t} \Phi-\Delta_{x} \Phi\right) d \tau\right) \\
& \quad+\int_{0}^{s} \exp \left(\int_{\tau}^{s}\left(\partial_{t} \Phi-\Delta_{x} \Phi\right) d \rho\right)\left(\partial_{t}-\Delta_{x}\right) a_{i-1} d \tau, \quad i \geq 1,
\end{aligned}
$$

where $a_{i 0}=\left.a_{i}\right|_{s=0}$ is a suitably chosen initial data, and $x=2 s p+y, y$ and $p$ being the initial data for the characteristic curve. Then in the region where the eikonal equation is solved, we have the estimate

$$
\left|\left(\partial_{t}-\Delta_{x}\right)\left(a e^{\lambda^{2} t+\lambda \Phi}\right)\right| \leq C \lambda^{-N} e^{\lambda^{2} t+\lambda \Phi} .
$$

For the later use, we shall compute $\partial_{t} \Phi_{2}(t, x)$ and $\Delta_{x} \Phi_{2}(t, x)$ along the segment with end points $y^{*}(t)$ and $z^{*}(t)$. We have by (4.19)

$$
\partial_{t} \Phi_{2}(t, x)=2 \partial_{t} s+\partial_{t} \varphi\left(t, y^{\prime}\right) .
$$

From (4.17) we have

$$
0=2\left(\partial_{t} s\right) p_{n}+2 s\left(\partial_{t} p_{n}\right)+\partial_{t} y_{n} .
$$

(4.16), $\left.\partial_{t} p_{n}\right|_{x^{\prime}=y^{*}(t)^{\prime}}=0$. Therefore,

$$
\left.2 \partial_{t} s\right|_{x^{\prime}=y^{*}(t)^{\prime}}=\left.\partial_{t} \varphi\left(t, y^{\prime}\right)\right|_{y^{\prime}=y^{*}(t)^{\prime}}=\frac{d}{d t} \varphi\left(t, y^{*}(t)^{\prime}\right)=\dot{a}(t) .
$$

Hence

$$
\left.\partial_{t} \Phi_{2}\right|_{x^{\prime}=y^{*}(t)^{\prime}}=2 \dot{a}(t) .
$$

Next we compute $\Delta_{x} \Phi_{2}(t, x)$. Let $k_{j}=k_{j}(t), 1 \leq j \leq n-1$, be the principal curvatures at $y^{*}(t)$. Without loss of generality, we assume that $y^{*}(t)=0$, and at $x=$ 0 , the directions of principal curvatures are equal to $(1,0, \cdots, 0), \cdots,(0, \cdots, 0,1,0)$. Then near $y^{*}(t), S_{\epsilon}(t)$ is written as

$$
x_{n}=-\frac{1}{2} \sum_{j=1}^{n-1} k_{j} x_{j}^{2}+O\left(\left|x^{\prime}\right|^{3}\right) .
$$

We parametrize $S_{\epsilon}(t)$ by $y^{\prime}=\left(y_{1}, \cdots, y_{n-1}\right)=\left(x_{1}, \cdots, x_{n-1}\right)$ and see that

$$
\left\{\begin{array}{l}
\partial_{i} \varphi=-k_{i} y_{i}+O\left(\left|y^{\prime}\right|^{2}\right), \quad 1 \leq i \leq n-1, \\
p_{i}=\partial_{i} \varphi\left(1-p_{n}\right)=-2 k_{i} y_{i}+O\left(\left|y^{\prime}\right|^{2}\right), \quad 1 \leq i \leq n-1, \\
p_{n}=\frac{\left|\nabla^{\prime} \varphi\right|^{2}-1}{\left|\nabla^{\prime} \varphi\right|^{2}+1}=-1+O\left(\left|y^{\prime}\right|^{2}\right) .
\end{array}\right.
$$


Using $x_{i}=2 s p_{i}+y_{i}$ and (4.33), we have when $y^{\prime}=0$,

$$
\left(\begin{array}{cccc}
\frac{\partial x_{1}}{\partial y_{1}} & \cdots & \frac{\partial x_{1}}{\partial y_{n-1}} & \frac{\partial x_{1}}{\partial s} \\
\cdots & \cdots & \cdots & \cdots \\
\cdots & \cdots & \cdots & \cdots \\
\frac{\partial x_{n}}{\partial y_{1}} & \cdots & \frac{\partial x_{n}}{\partial y_{n-1}} & \frac{\partial x_{n}}{\partial s}
\end{array}\right)=\left(\begin{array}{cccc}
1-4 s k_{1} & 0 & \cdots & 0 \\
\cdots & \cdots & \cdots & \cdots \\
0 & \cdots & 1-4 s k_{n-1} & 0 \\
0 & \cdots & 0 & -2
\end{array}\right)
$$

We also see that, when $y^{\prime}=0$,

$$
\frac{\partial p_{i}}{\partial x_{i}}=\sum_{j=1}^{n-1} \frac{\partial p_{i}}{\partial y_{j}} \frac{\partial y_{j}}{\partial x_{i}}+\frac{\partial p_{i}}{\partial s} \frac{\partial s}{\partial x_{i}}=\left\{\begin{aligned}
-2 k_{i} \frac{\partial y_{i}}{\partial x_{i}}, & 1 \leq i \leq n-1, \\
0, & i=n .
\end{aligned}\right.
$$

Therefore, we have, when $y^{\prime}=0$,

$$
\Delta_{x} \Phi_{2}=\nabla_{x} \cdot p=-\sum_{i=1}^{n-1} \frac{2 k_{i}}{1-4 s k_{i}} .
$$

By (4.32) and (4.36), we have proven the following lemma.

Lemma 4.4. Along the segment with end points $y^{*}(t)$ and $z^{*}(t)$, we have

$$
\exp \left(\int_{0}^{s}\left(\partial_{t} \Phi_{2}-\Delta_{x} \Phi_{2}\right) d \tau\right)=\frac{e^{2 s \dot{a}(t)}}{\prod_{j=1}^{n-1}\left(1-4 s k_{j}\right)^{1 / 2}} .
$$

\section{Approximate solutions}

5.1. Ansatz. We first construct an approximate solution $v_{a p r}(t, x ; \lambda)$ to $(1.1)$. Let us assume without loss of generality that $\mathbf{e}^{*}=\mathbf{e}_{\mathbf{n}}$. We take $\epsilon>0$ small enough and put $a_{1}(t), a_{2}(t)$ as follows:

$$
a_{1}(t)=a(t)-\epsilon^{3}, \quad a_{2}(t)=a(t)-\epsilon^{3} / 2 .
$$

We then have, letting $\partial S_{\epsilon}(t)$ be the boundary of $S_{\epsilon}(t)$,

$$
\left\{x \in S(t) ; a_{2}(t) \leq x_{n}\right\} \subset S_{\epsilon}(t), \quad \partial S_{\epsilon}(t) \subset\left\{x \in \Omega ; x_{n}<a_{1}(t)\right\}, \quad 0 \leq t \leq T .
$$

Our ansatz is the following form :

$$
\begin{gathered}
v_{a p r}(t, x ; \lambda)=b_{1}(t, x) \cdot \begin{cases}v_{+}(t, x ; \lambda) & \text { on } \quad \Omega \backslash D(t), \\
v_{-}(t, x ; \lambda) & \text { on } D(t),\end{cases} \\
v_{+}(t, x ; \lambda)=h_{\mathrm{fW}}(t, x ; \lambda)+\tilde{\chi}_{\epsilon}\left(\left|x^{\prime}-y^{*}(t)^{\prime}\right|\right) \sum_{j=2,3} a_{j}(t, x ; \lambda) e^{\lambda^{2} t+\lambda \Phi_{j}(t, x)}, \\
v_{-}(t, x ; \lambda)=a_{4}(t, x ; \lambda) e^{\lambda^{2} t+\lambda \Phi_{4}(t, x)},
\end{gathered}
$$

where $\Phi_{j}(t, x)$ 's are as in $\S 4$,

$$
b_{1}(t, x)=\chi_{\epsilon}\left(x_{n}-a(t)\right)
$$

with $\chi_{\epsilon}(\tau) \in C^{\infty}(\mathbb{R})$ satisfying $\chi_{\epsilon}(\tau)=0\left(\tau<-\epsilon^{3}\right), \chi_{\epsilon}(\tau)=1\left(\tau>-\epsilon^{3} / 2\right)$, and $\tilde{\chi}_{\epsilon} \in C^{\infty}(\mathbb{R})$ is such that $\tilde{\chi}_{\epsilon}(\tau)=1$ for $|\tau|<\epsilon / 2, \tilde{\chi}_{\epsilon}(\tau)=0$ for $|\tau|>\epsilon$. The 
following conditions are imposed:

$$
\begin{gathered}
v_{+}(t, x ; \lambda)=h_{\mathrm{fw}}(t, x ; \lambda) \quad \text { on } \quad \Gamma \cap\left\{x_{n}>a_{2}(t)\right\}, \\
v_{+}(t, x ; \lambda)=v_{-}(t, x ; \lambda) \quad \text { on } \quad S(t) .
\end{gathered}
$$

Note that choosing $\epsilon$ small enough, we can assume that

$$
\nu_{S(t)}(x) \cdot \mathbf{e}_{\mathbf{n}}>0 \quad \text { on } \quad S_{\epsilon}(t) .
$$

The intuition for this ansatz is as follows. We first give the heat flow $h_{\mathrm{fw}}(t, x ; \lambda)=$ $e^{\lambda^{2} t+\lambda x_{n}}$ from the boundary $\Gamma$. It is reflected at the inner boundary $S(t)$, giving rise to $e^{\lambda^{2} t+\lambda \Phi_{2}}$, which goes back to $\Gamma$. It is again reflected at $\Gamma$, and is transformed to $e^{\lambda^{2} t+\lambda \Phi_{3}}$. The heat flow $h_{\mathrm{fW}}(t, x ; \lambda)$ is also transmitted at the inner boundary, giving rise to $e^{\lambda^{2} t+\lambda \Phi_{4}}$.

We construct $a_{j}(t, x ; \lambda)$ taking into account of (4.29). We assume that $S_{\epsilon}(t)$ is parametrized as $y_{n}=\varphi\left(t, y^{\prime}\right)$, and let $s$ be the parameter along the associated caracteristic curve. We put, taking $N$ large enough, for $x \in D_{\epsilon,+}(t)$,

$$
\begin{aligned}
a_{2}(t, x ; \lambda)= & \sum_{i=0}^{N} \lambda^{-i} a_{2 i}(t, x), \\
a_{20}(t, x)= & a_{200}\left(t, y^{\prime}\right) \exp \left(\int_{0}^{s}\left(\partial_{t} \Phi_{2}-\Delta_{x} \Phi_{2}\right) d \tau\right), \\
a_{2 i}(t, x)= & a_{2 i 0}\left(t, y^{\prime}\right) \exp \left(\int_{0}^{s}\left(\partial_{t} \Phi_{2}-\Delta_{x} \Phi_{2}\right) d \tau\right) \\
& +\int_{0}^{s} \exp \left(\int_{\tau}^{s}\left(\partial_{t} \Phi_{2}-\Delta_{x} \Phi_{2}\right) d \rho\right)\left(\partial_{t}-\Delta_{x}\right) a_{2 i-1} d \tau, \quad i \geq 1,
\end{aligned}
$$

where $y \in S_{\epsilon}(t)$ is defined by (4.15), and $a_{2 i 0}\left(t, y^{\prime}\right)$ is to be determined later.

Observing that, on one hand, thanks to (4.14), $\Phi_{2}(t, x)<a(t)-\epsilon / 2$ if $\mid x^{\prime}-$ $y^{*}(t)^{\prime} \mid>\epsilon / 2$, and that, on the other hand, $\widetilde{\chi}_{\epsilon}\left(\left|x^{\prime}-y^{*}(t)^{\prime}\right|\right) \equiv 1$ for $\left|x^{\prime}-y^{*}(t)^{\prime}\right|<\epsilon / 2$, we can apply (4.30) to obtain

$$
\left|\left(\partial_{t}-\Delta_{x}\right)\left(a_{2} e^{\lambda^{2} t+\lambda \Phi_{2}(t, x)}\right)\right| \leq C \lambda^{-N} e^{\lambda^{2} t+\lambda a(t)} \quad \text { in } \quad D_{\epsilon,+}(t) .
$$

Similarly, assuming that $\Gamma_{\epsilon}(t)$ is written as $x_{n}=\widetilde{\varphi}\left(z^{\prime}\right)$ or $s=0$, where $s$ is the parameter along the associated characteristic curve with respect to $\Phi_{3}$, we put

$$
\begin{aligned}
a_{3}(t, x ; \lambda) & =\widetilde{\chi}_{\epsilon}(\bar{s}) \sum_{i=0}^{N} \lambda^{-i} a_{3 i}(t, x), \\
a_{30}(t, x) & =a_{300}\left(t, z^{\prime}\right) \exp \left(\int_{0}^{\bar{s}}\left(\partial_{t} \Phi_{3}-\Delta_{x} \Phi_{3}\right) d \tau\right) \\
a_{3 i}(t, x)= & a_{3 i 0}\left(t, y^{\prime}\right) \exp \left(\int_{0}^{\bar{s}}\left(\partial_{t} \Phi_{3}-\Delta_{x} \Phi_{3}\right) d \tau\right) \\
& \quad+\int_{0}^{\bar{s}} \exp \left(\int_{\tau}^{\bar{s}}\left(\partial_{t} \Phi_{3}-\Delta_{x} \Phi_{3}\right) d \rho\right)\left(\partial_{t}-\Delta_{x}\right) a_{3 i-1} d \tau, \quad i \geq 1,
\end{aligned}
$$


where $z \in \Gamma_{\epsilon}$ is defined by (4.23). Since $\Phi_{3}$ is strictly decreasing along the characteristic curve, we have, for $\epsilon>|s|>\epsilon / 2$,

$$
\Phi_{3} \leq\left.\Phi_{3}\right|_{\bar{s}=0}-\delta \leq\left.\Phi_{2}\right|_{\bar{s}=0}-\delta \leq a(t)-\delta
$$

for some $\delta>0$. For $|\bar{s}|<\epsilon / 2$, we have $\widetilde{\chi}_{\epsilon}(s) \equiv 1$, and so we can apply (4.30). Hence

$$
\left|\left(\partial_{t}-\Delta_{x}\right)\left(a_{3} e^{\lambda^{2} t+\lambda \Phi_{3}(t, x)}\right)\right| \leq C \lambda^{-N} e^{\lambda^{2} t+\lambda a(t)}, \quad \text { in } \quad D_{\epsilon,+}(t) .
$$

Finally we construct $a_{4}(t, x ; \lambda), x \in D(t)$, in a way similar to $a_{2}$. We put

$$
\begin{aligned}
a_{4}(t, x ; \lambda)= & \sum_{i=0}^{N} \lambda^{-i} a_{4 i}(t, x), \\
a_{40}(t, x)= & a_{400}\left(t, y^{\prime}\right) \exp \left(\int_{0}^{s}\left(\partial_{t} \Phi_{4}-k^{2} \Delta_{x} \Phi_{4}\right) d \tau\right), \\
a_{4 i}(t, x)= & a_{4 i 0}\left(t, y^{\prime}\right) \exp \left(\int_{0}^{s}\left(\partial_{t} \Phi_{4}-k^{2} \Delta_{x} \Phi_{4}\right) d \tau\right) \\
& +\int_{0}^{s} \exp \left(\int_{\tau}^{s}\left(\partial_{t} \Phi_{4}-k^{2} \Delta_{x} \Phi_{4}\right) d \rho\right)\left(\partial_{t}-k^{2} \Delta_{x}\right) a_{4 i-1} d \tau, \quad i \geq 1,
\end{aligned}
$$

where $s$ is the parameter along the associated characteristic curve in $D(t)$. Note that for small $\epsilon>0$,

$$
\left|\left(\partial_{t}-k^{2} \Delta_{x}\right)\left(a_{4} e^{\lambda^{2} t+\lambda \Phi_{4}(t, x)}\right)\right| \leq C \lambda^{-N} e^{\lambda^{2} t+\lambda a(t)},
$$

and $\chi_{\epsilon}=1$ near $y^{*}(t)$. Therefore, in the region where $\chi_{\epsilon}^{\prime} \neq 0, \Phi_{4} \leq a(t)-\delta$ for some $\delta>0$.

5.2. Matching. We determine $a_{j i 0}=\left.a_{j i}\right|_{s=0}$ in the following way. We put

$$
a_{1 i}(t, x)=\delta_{1 i},
$$

which implies

$$
h_{\mathrm{fW}}(t, x ; \lambda)=\sum_{i=0}^{N} \lambda^{-i} a_{1 i}(t, x) e^{\lambda^{2} t+\lambda \Phi_{1}(t, x)} .
$$

Granting that $a_{2 i 0}$ is given, $a_{3 i 0}$ and $a_{4 i 0}$ are defined by

$$
\begin{gathered}
a_{3 i 0}\left(t, z^{\prime}\right)=-a_{2 i}(t, x) \quad \text { on } \quad \Gamma_{\epsilon}(t), \\
a_{4 i 0}\left(t, y^{\prime}\right)=1+a_{2 i 0}\left(t, y^{\prime}\right) \quad \text { on } \quad S_{\epsilon}(t) .
\end{gathered}
$$

Since $\Phi_{2}(t, x)=\Phi_{3}(t, x)$ on $\Gamma_{\epsilon}(t),(5.14)$ implies (5.5). Since $\Phi_{2}(t, x)=\Phi_{4}(t, x)=$ $x_{n}$ on $S_{\epsilon}(t),(5.15)$ yields (5.6).

The above construction makes $v_{a p r}$ continuous. The jump of the derivative at $S(t)$ is computed as follows. For $x \in S(t)$ with $x_{n}<a(t)-\epsilon^{3} / 2$ we have

$$
\left|e^{-\lambda^{2} t-\lambda a(t)}\left[\gamma \partial_{\nu} v_{a p r}\right]_{S(t)}\right| \leq C \lambda e^{-\lambda \epsilon^{3} / 2} .
$$


For $x \in S(t)$ with $x_{n}>a(t)-\epsilon^{3} / 2$ we have $b_{1}(t, x) \equiv 1, x \in S_{\epsilon}(t)$, and so

$$
\begin{aligned}
& e^{-\lambda^{2} t-\lambda x_{n}}\left[\gamma \partial_{\nu} v_{a p r}\right]_{S(t)} \\
& =\lambda\left(a_{10} \nu_{n}+a_{20} \partial_{\nu} \Phi_{2}-k^{2} a_{40} \partial_{\nu} \Phi_{4}\right) \\
& +\sum_{i=0}^{N-1} \lambda^{-i}\left(a_{1 i+1} \nu_{n}+a_{2 i+1} \partial_{\nu} \Phi_{2}+\partial_{\nu} a_{1 i}+\partial_{\nu} a_{2 i}-a_{4 i+1} k^{2} \partial_{\nu} \Phi_{4}-k^{2} \partial_{\nu} a_{4 i}\right) \\
& +\lambda^{-N}\left(\partial_{\nu} a_{1 N}+\partial_{\nu} a_{2 N}-k^{2} a_{4 N}\right),
\end{aligned}
$$

where $\nu_{n}=\partial_{\nu} x_{n}$ is the $n$-th component of $\nu\left(t, x^{\prime}\right)$. The coefficients of the power $\lambda^{-i},-1 \leq i \leq N-1$, should vanish. Plugging the resulting equations with (5.15), we find the formula for $a_{200}$ :

$$
a_{200}\left(t, x^{\prime}\right)=-\left(\frac{\nu_{n}-k^{2} \partial_{\nu} \Phi_{4}}{\partial_{\nu} \Phi_{2}-k^{2} \partial_{\nu} \Phi_{4}}\right), \quad x \in S_{\epsilon} .
$$

Observing that

$$
\begin{aligned}
& \partial_{\nu} a_{2 i}=a_{2 i 0} \partial_{\nu}\left(\partial_{t} \Phi_{2}-\Delta \Phi_{2}\right), \quad \forall 0 \leq i \leq N, \quad x \in S_{\epsilon}, \\
& \partial_{\nu} a_{4 i}=\left(\delta_{1 i}+a_{2 i 0}\right) \partial_{\nu}\left(\partial_{t} \Phi_{4}-k^{2} \Delta \Phi_{4}\right), \quad \forall 0 \leq i \leq N, \quad x \in S_{\epsilon},
\end{aligned}
$$

we then obtain, for all $i \in[1, N]$, for all $x \in S_{\epsilon}$,

$$
\begin{aligned}
a_{2 i 0}= & \frac{\left(-\nu_{n}+k^{2} \partial_{\nu} \Phi_{4}\right) a_{1 i-1}-\partial_{\nu} a_{1 i-1}-\partial_{\nu} a_{2 i-1}+k^{2} \partial_{\nu} a_{4 i-1}}{\partial_{\nu} \Phi_{2}-k^{2} \partial_{\nu} \Phi_{4}} \\
= & \left(\partial_{\nu} \Phi_{2}-k^{2} \partial_{\nu} \Phi_{4}\right)^{-1}\left(\left(-\nu_{n}+k^{2} \partial_{\nu} \Phi_{4}+k^{2} \partial_{\nu}\left(\partial_{t} \Phi_{4}-k^{2} \Delta \Phi_{4}\right)\right) a_{1 i-1}\right. \\
& \left.-\partial_{\nu} a_{1 i-1}+\partial_{\nu}\left(k^{2}\left(\partial_{t} \Phi_{4}-k^{2} \Delta \Phi_{4}\right)-\left(\partial_{t} \Phi_{2}-\Delta \Phi_{2}\right)\right) a_{2 i-10}\right) .
\end{aligned}
$$

Then we have

$$
\left|\left[\gamma \partial_{\nu} v_{a p r}\right]_{S(t)}\right| \leq C \lambda^{-N} e^{\lambda^{2} t+\lambda a(t)} .
$$

Let us summarize the properties of $v_{a p r}(t, x ; \lambda)$. Let $\chi_{+}(t, x)$ be the characteristic function of $\Omega \backslash D(t)$, and put

$$
F(t, x ; \lambda)=\left(\frac{\partial}{\partial t}-\gamma(t, x) \Delta_{x}\right)\left(v_{a p r}(t, x ; \lambda)-\chi_{\epsilon}\left(x_{n}-a(t)\right) h_{\mathrm{fW}}(t, x ; \lambda) \chi_{+}(t, x)\right) .
$$

Lemma 5.1. (1) $v_{a p r} \in C([0, T] \times \Omega) \cap C^{\infty}\left(\overline{\mathcal{D}_{+}}\right) \cap C^{\infty}\left(\overline{\mathcal{D}_{-}}\right)$, in particular

$$
\left[v_{a p r}\right]_{S(t)}=0 \text {. }
$$

(2) There exists a constant $C>0$ independent of $t, x, \lambda$ such that

$$
\left|\left[\gamma \partial_{\nu} v_{a p r}\right]_{S(t)}\right| \leq C \lambda^{-N} e^{\lambda^{2} t+\lambda a(t)} .
$$

(3a) At $y=y^{*}(t)$,

$$
a_{200}\left(t, y^{*}(t)^{\prime}\right)=\frac{1-k}{1+k}
$$


(3b) At $x^{\prime}=0$,

$$
a_{20}(t, x)=\frac{1-k}{1+k} \frac{e^{2 s \dot{a}(t)}}{\prod_{j=1}^{n-1}\left(1-4 s k_{j}\right)^{1 / 2}} .
$$

(4) There exists a constant $C>0$ independent of $t, x, \lambda$ such that

$$
|F(t, x ; \lambda)| \leq C \lambda^{-N} e^{\lambda^{2} t+\lambda a(t)} .
$$

Proof. Everything has already been proved except for (3), which follows from (5.16), from the form of $p_{j}{ }^{\prime} \mathrm{s}$ in Lemmas 4.1 and 4.3, and from Lemma 4.4.

5.3. The function $H$. We next construct the function $H$ in Theorem 3.1. The idea is the same as above, however it must be $C^{1}$ across $S_{\epsilon}(t)$ with the trade off that it merely satisfies the differential inequality (3.9). Letting $\chi_{+}(t, x)$ and $\chi_{-}(t, x)$ be the characteristic functions of $\Omega \backslash D(t)$ and $D(t)$, we construct $H=H(t, x ; \lambda)$ in the following form:

$$
\begin{aligned}
H(t, x ; \lambda) & =e^{-\lambda^{2} t+\lambda a_{2}(t)} \\
& +b_{1}(t, x) \chi_{+}(t, x) e^{-\lambda^{2} t}\left(e^{\lambda x_{n}}+b_{2}(t, x ; \lambda) e^{\lambda \Phi_{2}(t, x)}\right) \\
& +b_{1}(t, x) \chi_{-}(t, x) b_{4}(t, x ; \lambda) e^{-\lambda^{2} t+\lambda \Phi_{4}(t, x)},
\end{aligned}
$$

where $a_{2}(t)$ is the function in (5.1). Let us construct the coefficients $b_{j}(j=2,4)$. Recall that $0 \leq b_{1}(t, x) \leq 1$ and

$$
b_{1}(t, x)=0, \quad \text { if } \quad x_{n}<a_{1}(t), \quad b_{1}(t, x)=1, \quad \text { if } \quad x_{n}>a_{2}(t) .
$$

We introduce the local coordinates $\left(\tau, y^{\prime}\right)$ around $S_{\epsilon}(t)$ by

$$
x=\tau \nu\left(t, y^{\prime}\right)+\left(y^{\prime}, \varphi\left(t, y^{\prime}\right)\right), \quad|\tau|<\epsilon^{3} .
$$

We are going to construct $b_{2}, b_{4}$ in the form

$$
b_{2}(t, x)=\chi(\tau) b_{20}\left(t, y^{\prime}\right), \quad b_{4}(t, x)=\chi(\tau) b_{40}\left(t, y^{\prime}\right),
$$

where $\chi(\tau) \in C^{\infty}(\mathbb{R})$ such that $0 \leq \chi(\tau) \leq 1, \chi(\tau)=1\left(|\tau|<\epsilon^{3} / 2\right), \chi(\tau)=$ $0\left(|\tau|>\epsilon^{3}\right)$. For $\epsilon>0$ chosen small enough, $x \rightarrow\left(\tau, y^{\prime}\right)$ is a diffeomorphism in the $\epsilon^{3}$-neighbourhood of $S_{\epsilon}(t)$. Then the conditions $[H]_{S(t)}=\left[\gamma \partial_{\nu} H\right]_{S(t)}=0$ are rewritten as

$$
\left\{\begin{aligned}
1+b_{20} & =b_{40}, \\
\nu_{n}+b_{20} \partial_{\nu} \Phi_{2} & =k^{2} b_{40} \partial_{\nu} \Phi_{4} .
\end{aligned}\right.
$$

Solving the above equation we get

$$
\begin{aligned}
& b_{20}=\frac{\nu_{n}-k^{2} \partial_{\nu} \Phi_{4}}{k^{2} \partial_{\nu} \Phi_{4}-\partial_{\nu} \Phi_{2}}, \\
& b_{40}=\frac{\nu_{n}-\partial_{\nu} \Phi_{2}}{k^{2} \partial_{\nu} \Phi_{4}-\partial_{\nu} \Phi_{2}} .
\end{aligned}
$$


Lemma 5.2. (1) $H(t, x ; \lambda) \in C^{\infty}\left(\overline{\mathcal{D}_{+}}\right) \cap C^{\infty}\left(\overline{\mathcal{D}_{-}}\right)$, and satisfies

$$
[H(t, x ; \lambda)]_{S(t)}=\left[\gamma \partial_{\nu} H(t, x ; \lambda)\right]_{S(t)}=0 .
$$

(2) There exists a constant $C>0$ independent of large $\lambda$ such that

$$
e^{\lambda^{2} t} H(t, x ; \lambda) \geq C\left\{\begin{array}{l}
e^{\lambda x_{n}}, \quad x \in \Omega \backslash D(t), \\
e^{\lambda \Phi_{4}(t, x)}, \quad x \in D(t) .
\end{array}\right.
$$

(3) Let $\chi_{S_{\epsilon}(t)}(x)$ be the characteristic function of the $\epsilon^{3}$-neighbourhood of $S_{\epsilon}(t)$.

Then there exists a constant $C>0$ independent of $\lambda$ such that

$$
e^{\lambda^{2} t} H(t, x ; \lambda) \leq e^{\lambda a_{2}(t)}+b_{1}(t, x) \cdot\left\{\begin{array}{l}
e^{\lambda x_{n}}+C \chi_{S_{\epsilon}(t)}(x) e^{\lambda \Phi_{2}(t, x)}, \quad x \in \Omega \backslash D(t), \\
C \chi_{S_{\epsilon}(t)}(x) e^{\lambda \Phi_{4}(t, x)}, \quad x \in D(t) .
\end{array}\right.
$$

(4) There exists a constant $\sigma>0$ independent of $\lambda$ such that

$$
\left(\partial_{t}+\gamma(t, x) \Delta_{x}\right) H(t, x ; \lambda) \leq \sigma \lambda H(t, x ; \lambda), \quad x \notin S(t) .
$$

(5) There exists a constant $C>0$ independent of $\lambda$ such that

$$
H(t, x ; \lambda) \leq e^{-\lambda^{2} t+\lambda a_{2}(t)}+C e^{-\lambda^{2} t+\lambda x_{n}} \quad \text { on } \quad S(t) .
$$

Proof. The assertion (1) follows from our construction. To prove (2), recall that Lemmas 4.1 and 4.3 imply $\Phi_{2}(t, x) \leq x_{n}$, and

$$
\partial_{\nu} \Phi_{2}=-1, \quad \partial_{\nu} \Phi_{4}=1 / k, \quad \text { at } \quad x=y^{*}(t) .
$$

We then have, near $y^{*}(t)$,

$$
e^{\lambda x_{n}}+b_{2} e^{\lambda \Phi_{2}} \geq e^{\lambda x_{n}}\left(1-\left|b_{2}\right|\right) .
$$

At $x=y^{*}(t)$, observing that

we then have

$$
b_{20}=\frac{1-k}{1+k}
$$

$$
1-\left|b_{2}\right|=1-\frac{|1-k|}{1+k}>0 \text {. }
$$

Then there exists a constant $C>0$ such that in a small neighbourhood of $y^{*}(t)$

$$
e^{\lambda x_{n}}+b_{2} e^{\lambda \Phi_{2}} \geq C e^{\lambda x_{n}}
$$

holds. This proves the first inequality of (2) in a small neighbourhood of $y^{*}(t)$. Outside of this neighbourhood, $\Phi_{2}(t, x)<x_{n}-\delta$ for some constant $\delta>0$. We take $a_{3}(t)$ so that $a_{1}(t)<a_{3}(t)<a_{2}(t)$ and $b_{1}\left(t, a_{3}(t)\right)>0$. Then if $x_{n} \geq a_{3}(t)$ and $x$ is outside the above mentioned neighbourhood, we have

$$
b_{1}(t, x)\left(e^{\lambda x_{n}}+b_{2} e^{\lambda \Phi_{2}}\right) \geq e^{\lambda x_{n}}\left(b_{1}\left(t, a_{3}(t)\right)-O\left(e^{-\lambda \delta}\right)\right) .
$$

Therefore the first inequality again holds if $x_{n} \geq a_{3}(t)$. If $x_{n}<a_{3}(t)$, recalling the term $e^{\lambda a_{2}(t)}$ of $H(t, x)$, we get the desired inequality. By a suitable choice of $\epsilon$, the second inequality holds for $x$ closed to $y^{*}(t), x \in D(t)$, since $b_{4}\left(t, y^{*}(t)\right)=\frac{2}{1+k}>0$. If $x_{n} \leq a_{3}(t)$, the desired inequality is obvious due to the term $e^{\lambda a_{2}(t)}$.

The estimate (3) is easy to prove. The estimate (5) is a consequence of (3). 
Since $\Phi_{j}$ 's satisfy the eikonal equations, we have

$$
\begin{aligned}
e^{\lambda^{2} t} & \left|\left(\partial_{t}+\gamma(t, x) \Delta_{x}\right) H(t, x)\right| \\
\leq C \lambda & \begin{cases}e^{\lambda a_{2}(t)}+e^{\lambda x_{n}}+\chi_{S_{\epsilon}(t)}(x) e^{\lambda \Phi_{2}(t, x)}, \quad x \in \Omega \backslash D(t), \quad x>a_{1}(t), \\
e^{\lambda a_{2}(t)}+\chi_{S_{\epsilon}(t)}(x) e^{\lambda \Phi_{2}(t, x)}, \quad x \in \Omega \backslash D(t), \quad x<a_{1}(t), \\
e^{\lambda a_{2}(t)}+\chi_{S_{\epsilon}(t)}(x) e^{\lambda \Phi_{4}(t, x)}, & x \in D(t) .\end{cases}
\end{aligned}
$$

The assertion (4) follows directly from (2) and this inequality.

5.4. The function $H_{\lambda, \mu}$. The function $H(t, x ; \lambda)$ has the desired properties except for the assertion (4) in Lemma 5.2, where $\sigma \lambda$ should be $-\sigma \lambda$. The remedy is as follows.

For a new parameter $\mu$, we put

$$
H_{\lambda, \mu}(t, x)=e^{-\lambda^{2} t+(\lambda-\mu)^{2} t} H(t, x ; \lambda-\mu) .
$$

This simple trick brings us the desired inequality.

Lemma 5.3. Let $\sigma>0$ be the constant in Lemma 5.2 (4).

(1) If $\mu>\sigma$ and $\lambda \geq \frac{\mu^{2}}{\mu-\sigma}$, we have

$$
\left(\partial_{t}+\gamma(t, x) \Delta_{x}\right) H_{\lambda, \mu}(t, x) \leq-\mu \lambda H_{\lambda, \mu}(t, x), \quad \text { in } \quad L^{2}\left(\mathcal{D}_{ \pm}\right) .
$$

(2) There exists a constant $C>0$ independent of large $\lambda$ and $\mu$ such that

$$
e^{\lambda^{2} t} H_{\lambda, \mu}(t, x) \geq C\left\{\begin{array}{c}
e^{(\lambda-\mu) x_{n}}, \quad x \in \Omega \backslash D(t), \\
e^{\lambda \Phi_{4}(t, x)}, \quad x \in D(t) .
\end{array}\right.
$$

(3) Let $\chi_{S_{\epsilon}(t)}(x)$ be the characteristic function of the $\delta$-neighbourhood of $S_{\epsilon}(t)$. Then there exists a constant $C>0$ independent of $\lambda$ such that

$$
\begin{gathered}
\quad e^{\lambda^{2} t} H_{\lambda, \mu}(t, x) \\
\leq e^{\lambda a_{2}(t)}+b_{1}(t, x) \cdot\left\{\begin{array}{l}
e^{(\lambda-\mu) x_{n}}+C \chi_{S_{\epsilon}(t)}(x) e^{(\lambda-\mu) \Phi_{2}(t, x)}, \quad x \in \Omega \backslash D(t), \\
C \chi_{S_{\epsilon}(t)}(x) e^{(\lambda-\mu) \Phi_{4}(t, x)}, \quad x \in D(t) .
\end{array}\right. \\
\text { (4) }\left[H_{\lambda, \mu}\right]_{S(t)}=\left[\gamma \partial_{\nu} H_{\lambda, \mu}\right]_{S(t)}=0 .
\end{gathered}
$$

(5) There exists a constant $C>0$ independent of $\lambda$ such that

$$
H_{\lambda, \mu}(t, x) \leq e^{-\lambda^{2} t+\lambda a_{2}(t)}+C e^{-\lambda^{2} t+\lambda x_{n}} \quad \text { on } \quad S(t) .
$$

Proof. By using Lemma 5.2 (4), we have

$$
\begin{aligned}
& \left(\partial_{t}+\gamma \Delta_{x}\right) H_{\lambda, \mu} \\
& =e^{-\lambda^{2} t+(\lambda-\mu)^{2} t}\left(\partial_{t}+\gamma \Delta_{x}\right) H(t, x ; \lambda-\mu)+\left(-2 \mu \lambda+\mu^{2}\right) H_{\lambda, \mu} \\
& \leq\left(\sigma \lambda-2 \mu \lambda+\mu^{2}\right) H_{\lambda, \mu},
\end{aligned}
$$

from which the assertion (1) follows immediately. The assertion (2) is a consequence of Lemma 5.2 (3). The assertions (3), (4) and (5) follow from the construction and Lemma 5.2 . 
Let $b_{1}(t, x)$ be the function appearing in (5.20). We set

$$
\widetilde{b}_{1}(t, x)=1-b_{1}(t, x) \in C^{\infty}([0, T] \times \bar{\Omega}) .
$$

Observe that

$$
\operatorname{supp} \widetilde{b}_{1}(t, x) \subset\left\{x_{n} \leq a_{2}(t)\right\}, \quad a_{2}(t) \leq a(t)-\delta,
$$

for $\delta=\epsilon^{3} / 2>0$.

We need the following

Lemma 5.4. Set

$$
G=\left(\partial_{t}-\gamma \Delta_{x}\right)\left(\widetilde{b}_{1}(t, x) h_{f w}(t, x ; \lambda)\right) .
$$

Then we have

$$
|G| \leq C \lambda^{2} e^{\lambda^{2} t+\lambda(a(t)-\delta)}
$$

where the constant $C$ is independent of $\lambda>0$.

Proof. It comes from

$$
\left|\partial_{t}\left(\widetilde{b}_{1}(t, x) e^{\lambda x_{n}}\right)\right|+\left|\left(\partial_{x}\right)^{\alpha}\left(\widetilde{b}_{1}(t, x) e^{\lambda x_{n}}\right)\right| \leq C_{\alpha} \lambda^{|\alpha|} e^{\lambda(a(t)-\delta)} .
$$

\section{BOUNDARY INTEGRALS}

6.1. Laplace's method. Let us recall the well-known Laplace's method for computation of integrals with large parameters. Let $\Sigma$ be a compact hypersurface in $\mathbb{R}^{n}$ and consider the integral with large parameter $\lambda>0$

$$
I(\lambda)=\int_{\Sigma} e^{\lambda \Phi(x)} A_{\lambda}(x) d \Sigma,
$$

where $d \Sigma$ is the surface element of $S, \Phi(x)$ and $A_{\lambda}(x)$ are smooth functions on $\Sigma$ and satisfy

$$
\left|\partial_{x}^{k} A_{\lambda}(x)\right| \leq C, \quad 0 \leq k \leq 3,
$$

for some constant $C>0$. We assume that $\Phi(x)$ is real-valued. Let $\Phi^{\prime \prime}(x)$ be the Hessian matrix of $\Phi$ at $x \in \Sigma$ with respect to local coordinates. We assume that

$\Phi(x)$ attains its maximum only at $p \in \Sigma$, and $\Phi^{\prime \prime}(p)$ is negative definite.

Let $U$ be a small neighbourhood of $p$ and $U^{\prime}=\Sigma \backslash U$. Then there exist constants $\epsilon, C>0$ such that

$$
\left|\int_{U^{\prime}} e^{\lambda \Phi(x)} A_{\lambda}(x) d \Sigma\right| \leq C e^{\lambda(\Phi(p)-\epsilon)} .
$$

We shall assume without loss of generality that, near $p, \Sigma$ is represented as $x_{n}=f\left(x^{\prime}\right), x^{\prime}=\left(x_{1}, \cdots, x_{n-1}\right)$, and $\Phi(x)$ takes the form

$$
\Phi(x)=\Phi(p)-\frac{1}{2} \sum_{i=1}^{n-1} \mu_{i}\left(x_{i}-p_{i}\right)^{2}+O\left(\left|x^{\prime}-p^{\prime}\right|^{3}\right),
$$


where $-\mu_{i}$ 's are eigenvalues of $\Phi^{\prime \prime}(p)$. By the Morse lemma (see e.g. [9], p. 502), there is a diffeomorphism $x^{\prime} \rightarrow \xi=\left(\xi_{1}, \cdots, \xi_{n-1}\right)$ such that

$$
\Phi(x)=\Phi(p)-\frac{1}{2} \sum_{i=1}^{n-1} \xi_{i}^{2}, \quad\left|\frac{\partial x^{\prime}}{\partial \xi}(0)\right|=\left|\operatorname{det} \Phi^{\prime \prime}(p)\right|^{-1 / 2} .
$$

Then we have, letting $\nabla^{\prime}=\left(\partial / \partial x_{1}, \cdots, \partial / \partial x_{n-1}\right)$,

$$
\begin{gathered}
\int_{U} e^{\lambda \Phi(x)} A_{\lambda}(x) d \Sigma=e^{\lambda \Phi(p)} \int_{\xi(U)} e^{-\frac{\lambda}{2}|\xi|^{2}} b_{\lambda}(\xi) d \xi, \\
b_{\lambda}(\xi)=A_{\lambda}(x)\left(1+\left|\nabla^{\prime} f\right|^{2}\right)^{1 / 2}\left|\frac{\partial x^{\prime}}{\partial \xi}\right|
\end{gathered}
$$

By the change of variable $\eta=\sqrt{\lambda / 2} \xi$, and the Taylor expansion, we then have

$$
\begin{aligned}
& \int_{\Sigma} e^{\lambda \Phi(x)} A_{\lambda}(x) d \Sigma \\
& =e^{\lambda \Phi(p)}\left(\frac{2 \pi}{\lambda}\right)^{(n-1) / 2}\left(\frac{1+\left|\nabla^{\prime} f(p)\right|^{2}}{\left|\operatorname{det} \Phi^{\prime \prime}(p)\right|}\right)^{1 / 2}\left(A_{\lambda}(p)+O\left(\frac{1}{\lambda}\right)\right) .
\end{aligned}
$$

Let us apply this result to our integral on the inner boundary, i.e. $\Phi(x)=2 x_{n}$ and $\Sigma=\overline{S_{\epsilon}(t)}$ or $S(t)$. Then we have $p=y^{*}(t), \Phi(p)=2 a(t),\left|\operatorname{det} \Phi^{\prime \prime}(p)\right|=$ $\Pi_{i=1}^{n-1} \mu_{i}(t), \nabla^{\prime} f(p)=\nabla^{\prime} \varphi\left(t, y^{*}\right)=0$. We then obtain the following asymptotic expansion which is uniform for $0 \leq t \leq T$ :

$$
\int_{S(t)} e^{2 \lambda x_{n}} A_{\lambda}(x) d S(t)=e^{2 \lambda a(t)}\left(\prod_{i=1}^{n-1} \frac{\pi}{\lambda \mu_{i}(t)}\right)^{1 / 2}\left(A_{\lambda}\left(y^{*}(t)\right)+O\left(\frac{1}{\lambda}\right)\right) .
$$

6.2. Estimates of boundary integrals. The following simple lemma is useful.

Lemma 6.1. There exists $C>0$ such that

$$
\begin{gathered}
\int_{0}^{T} e^{\lambda(\mu t+2 a(t))} d t \leq C \frac{e^{\lambda(\mu T+2 a(T))}}{\lambda}, \quad \text { if } \quad \lambda>0, \quad \mu>4 \max _{0 \leq t \leq T}\left|a^{\prime}(t)\right|, \\
\int_{0}^{T} e^{\lambda^{2} t+\lambda a(t)} d t \leq C \frac{e^{\lambda^{2} T+\lambda a(T)}}{\lambda^{2}}, \quad \text { if } \quad \lambda>2 \max _{0 \leq t \leq T}\left|a^{\prime}(t)\right| .
\end{gathered}
$$

Moreover, let $f_{\lambda}(t)$ be a smooth function on $[0, T]$ such that

$$
\left|f_{\lambda}(t)\right|+\left|\frac{d}{d t} f_{\lambda}(t)\right| \leq C
$$

for some constant $C>0$. Then, if $\mu>4 \max _{0 \leq t \leq T}\left|a^{\prime}(t)\right|$, we have

$$
\int_{0}^{T} e^{\lambda(\mu t+2 a(t))} f_{\lambda}(t) d t=\frac{e^{\lambda(\mu T+2 a(T))}}{\lambda(\mu+2 \dot{a}(T))}\left(f_{\lambda}(T)+O\left(\frac{1}{\lambda}\right)\right) .
$$

Proof. We use the change of variable $\rho=t+2 a(t) / \mu, \rho_{\min }=\rho(0), \rho_{\max }=\rho(T)$ to see that

$$
I\left(\lambda, f_{\lambda}\right):=\int_{0}^{T} e^{\lambda(\mu t+2 a(t))} f_{\lambda}(t) d t=\int_{\rho_{\min }}^{\rho_{\max }} e^{\lambda \mu \rho} \frac{d t}{d \rho} f_{\lambda}(t(\rho)) d \rho .
$$


By using (6.7) we obtain (6.5). The proof of (6.6) is similar. By using (6.7) we can write

$$
f_{\lambda}(t)=f_{\lambda}(T)+(T-t) g_{\lambda}(t)
$$

where $\left|g_{\lambda}\right| \leq C$. Hence

$$
I\left(\lambda, f_{\lambda}\right)=f_{\lambda}(T) I(\lambda, 1)+I\left(\lambda,(T-t) g_{\lambda}\right) .
$$

We then have

$$
\left|I\left(\lambda,(T-t) g_{\lambda}\right)\right| \leq C I(\lambda,(T-t)) \leq C^{\prime} \int_{\rho_{\min }}^{\rho_{\max }}\left(\rho_{\max }-\rho\right) e^{\lambda \mu \rho} d \rho \leq C \frac{e^{\lambda \rho_{\max }}}{\lambda^{2}},
$$

which proves (6.8).

We split the above solution $u$ into two parts

$$
u=v+w,
$$

where $v$ and $w$ satisfy

$(6.9)\left\{\begin{aligned} \partial_{t} v-\nabla \cdot(\gamma \nabla v) & =0 \text { in }\left(T_{0}, T\right) \times \Omega, \\ v & =b_{1}(\cdot) h_{\mathrm{fw}}(\cdot ; \lambda) \text { on }\left(T_{0}, T\right) \times \partial \Omega, \\ \left.v\right|_{t=T_{0}} & =u\left(T_{0}, \cdot ; \lambda\right)+b_{1}\left(T_{0}, \cdot\right) h_{\mathrm{fw}}\left(T_{0}, \cdot ; \lambda\right) \text { on } \Omega,\end{aligned}\right.$

$$
\left\{\begin{aligned}
\partial_{t} w-\nabla \cdot(\gamma \nabla w) & =0 \text { in }(0, T) \times \Omega \\
w & =\widetilde{b}_{1}(\cdot) h_{\mathrm{fw}}(\cdot ; \lambda) \text { on }(0, T) \times \partial \Omega \\
\left.w\right|_{t=0} & =\widetilde{b}_{1}(0, \cdot) h_{\mathrm{fw}}(0, \cdot ; \lambda) \quad \text { on } \Omega
\end{aligned}\right.
$$

where $b_{1}$ and $\widetilde{b}_{1}$ are as in (5.20) and (5.26).

Lemma 6.2. Let $v_{a p r}(t, x ; \lambda)$ be as in Lemma 5.1. Then, there exists $\mu_{0}>0$ depending only on $\Omega$ such that, letting $\mu>\mu_{0}$, there exists $\lambda_{0}>0$ so that for all $\lambda>\lambda_{0}$ we have

$$
\begin{aligned}
& \int_{T_{0}}^{T} e^{\lambda \mu t} d t \int_{\Gamma} h_{b w}(t, x ; \lambda)\left|\partial_{\nu}\left(v(t, x ; \lambda)-v_{a p r}(t, x ; \lambda)\right)\right| d \Gamma \\
& \leq C \lambda^{-N-(n+1) / 2} e^{\lambda(\mu T+2 a(T))}
\end{aligned}
$$

Proof. Letting $V=v-v_{a p r}$, we have

$$
\left\{\begin{aligned}
\partial_{t} V-\nabla \cdot(\gamma \nabla V) & =F \quad \text { in } \quad L^{2}\left(\mathcal{D}_{ \pm}\right), \\
V & =0 \quad \text { on } \quad\left(T_{0}, T\right) \times \partial \Omega \\
\left.V\right|_{t=T_{0}} & =u\left(T_{0}, \cdot ; \lambda\right)+b_{1}\left(T_{0}, \cdot\right) h_{\mathrm{fw}}\left(T_{0}, \cdot ; \lambda\right)-\left.v_{a p r}\right|_{t=T_{0}} \quad \text { on } \quad \Omega,
\end{aligned}\right.
$$

where $F$ satisfies (5.18). We apply Theorem 3.1 in the cylinder $\left(T_{0}, T\right) \times \Omega$ with $H=H_{\lambda, \mu}(t, x)$. By Lemma 5.1 (2) and Lemma 5.3 (5), we have

$$
\left|\left[\gamma \partial_{\nu} v_{a p r}\right]_{S(t)} H_{\lambda, \mu}\right| \leq C \lambda^{-N}\left(e^{\lambda\left(a(t)+a_{2}(t)\right)}+e^{\lambda\left(a(t)+x_{n}\right)}\right) .
$$


Therefore, we have

$$
\begin{aligned}
& e^{\lambda \mu T} E\left(V, H_{\lambda, \mu} ; T\right)+\int_{T_{0}}^{T} e^{\lambda \mu t} E_{\Gamma}\left(\partial_{\nu} V, H_{\lambda, \mu} ; t\right) d t \\
& \leq E\left(V, H_{\lambda, \mu} ; T_{0}\right)+C \lambda^{-N} \int_{T_{0}}^{T} e^{\lambda \mu t} d t \int_{S(t)}\left(e^{\lambda(2 a(t)-\delta)}+e^{\lambda\left(a(t)+x_{n}\right)}\right) d S(t),
\end{aligned}
$$

for some $\delta>0$. Thanks to Lemmas 3.3, $5.3(3)$, and observing that

$$
\left|v_{a p r}(\cdot ; \lambda)\right|+H_{\lambda, \mu} \leq C e^{\lambda^{2} t+\lambda \rho_{0}} \quad \text { in } \quad(0, T) \times \Omega
$$

for some $\rho_{0}$ depending only on $\Omega$, we have

$$
E\left(V, H_{\lambda, \mu} ; T_{0}\right) \leq\left\|V\left(T_{0}\right)\right\|_{L^{1}(\Omega)} \sup _{\Omega} H_{\lambda, \mu}\left(T_{0}\right) \leq C e^{\lambda \rho}
$$

for some $\rho$ depending only on $\Omega$. We apply (6.4) to see that

$$
\int_{S(t)} e^{\lambda x_{n}} d S(t) \leq C \lambda^{-(n-1) / 2} e^{\lambda a(t)}
$$

Next we apply (6.5) to obtain

$$
\int_{0}^{T} e^{\lambda \mu t} d t \int_{S(t)} e^{\lambda\left(a(t)+x_{n}\right)} d S(t) \leq C \lambda^{-(n+1) / 2} e^{\lambda(\mu T+2 a(T))}
$$

Therefore, we have

$$
\begin{aligned}
& \int_{T_{0}}^{T} e^{\lambda \mu t} d t \int_{\Gamma} H_{\lambda, \mu}(t, x)\left|\partial_{\nu}\left(v-v_{a p r}\right)\right| d \Gamma \\
\leq & C\left(e^{\lambda \rho}+e^{\lambda(\mu T+2 a(T)-\delta)}+\lambda^{-N-(n+1) / 2} e^{\lambda(\mu T+2 a(T))}\right) .
\end{aligned}
$$

If $\mu>T^{-1}\left(\rho-2 \inf \left\{x_{n} ; x \in \Omega\right\}\right)$, then $\mu T+2 a(T)>\rho$ and so

$$
\int_{T_{0}}^{T} e^{\lambda \mu t} d t \int_{\Gamma} H_{\lambda, \mu}(t, x)\left|\partial_{\nu}\left(v-v_{a p r}\right)\right| d \Gamma \leq C \lambda^{-N-(n+1) / 2} e^{\lambda(\mu T+2 a(T))} .
$$

We have, by using Lemma $5.3(2)$,

$$
0<h_{\mathrm{bW}}(t, x ; \lambda) \leq C H_{\lambda, \mu}(t, x), \quad \text { on } \quad(0, T) \times \Gamma .
$$

This, together with $\mu_{0}>T^{-1}\left(\rho-2 \inf \left\{x_{n} ; x \in \Omega\right\}\right)$ and (6.11), proves the lemma.

Lemma 6.3. We have

$$
\begin{aligned}
\int_{0}^{T} e^{\lambda \mu t} d t & \int_{\Gamma} h_{b w}(t, x ; \lambda)\left|\partial_{\nu}\left(w(t, x ; \lambda)-\widetilde{b}_{1}(t, x) h_{f w}(t, x ; \lambda)\right)\right| d \Gamma \\
& \leq C \lambda^{-N-(n+1) / 2} e^{\lambda(\mu T+2 a(T))}
\end{aligned}
$$

Proof. We put $W=w-\widetilde{b}_{1} h_{\mathrm{fw}}(\cdot ; \lambda)$. Then we have the equation

$$
\left\{\begin{aligned}
\partial_{t} W-\nabla \cdot(\gamma \nabla W) & =-G \quad \text { in } \quad L^{2}\left(\mathcal{D}_{ \pm}\right) \\
W & =0 \quad \text { on } \quad(0, T) \times \partial \Omega \\
\left.W\right|_{t=0} & =0 \quad \text { on } \quad \Omega
\end{aligned}\right.
$$


where $G$ is defined by (5.27). We apply Theorem 3.1 with $H=H_{\lambda, \mu}$ and use Lemma 5.4 to obtain the lemma.

\section{Theorem 6.4.}

$$
\begin{gathered}
\mid \int_{T_{0}}^{T} e^{\lambda \mu t} d t \int_{\Gamma} h_{b w}(t, x ; \lambda) \partial_{\nu}\left(u(t, x ; \lambda)-h_{f w}(t, x ; \lambda)\right. \\
\left.-\sum_{j=1,2} a_{j}(t, x) e^{\lambda^{2} t+\lambda \Phi_{j}(t, x)}\right) d \Gamma \mid \\
\leq C \lambda^{-N-(n+1) / 2} e^{\lambda(\mu T+2 a(T))}
\end{gathered}
$$

Proof. Lemmas 6.2 and 6.3 imply

$$
\begin{gathered}
\mid \int_{T_{0}}^{T} e^{\lambda \mu t} d t \int_{\Gamma} h_{\mathrm{bW}}(t, x ; \lambda) \partial_{\nu}\left(u(t, x ; \lambda)-b_{1}(t, x) h_{\mathrm{fW}}(t, x ; \lambda)\right. \\
\left.\quad-\sum_{j=1,2} a_{j}(t, x) e^{\lambda^{2} t+\lambda \Phi_{j}(t, x)}\right) d \Gamma \mid \\
\leq C \lambda^{-N-(n+1) / 2} e^{\lambda(\mu T+2 a(T))} .
\end{gathered}
$$

Using (5.20), one can replace $b_{1}(t, x)$ by 1 to prove the theorem.

\section{Proof of Main theOrems}

7.1. Proof of Theorem 1.1. As above, we assume that $\mathbf{e}^{*}=\mathbf{e}_{\mathbf{n}}$, and denote $p \in \mathbb{R}^{n}$ by $p=\left(p^{\prime}, p_{n}\right), p^{\prime}=\left(p_{1}, \cdots, p_{n-1}\right)$. We fix $t$ and, by suitable rotation and translation, take the rectangular coordinates of $\mathbb{R}^{n}$ so that $y^{*}(t)=(0, a(t))$ and in a small neighborhood of $y^{*}(t)$, which is denoted by $U(t), S(t)$ is represented as $y_{n}=\varphi\left(t, y^{\prime}\right)$, moreover

$$
\varphi\left(t, y^{\prime}\right)=a(t)-\frac{1}{2} \sum_{i=1}^{n-1} k_{i}(t) y_{i}^{2}+O\left(\left|y^{\prime}\right|^{3}\right),
$$

where $k_{i}(t)$ 's are the principal curvatures of $S(t)$ at $y^{*}(t)$. Recall that the characteristic curve $X\left(t, s, y^{\prime}\right)$ is written as

$$
X\left(t, s, y^{\prime}\right)=2 s p\left(t, y^{\prime}\right)+y \text {. }
$$

By (4.33),

$$
p_{i}\left(t, y^{\prime}\right)=\left\{\begin{array}{l}
-2 k_{i}(t) y_{i}+O\left(\left|y^{\prime}\right|^{2}\right), \quad i \neq n, \\
-1+O\left(\left|y^{\prime}\right|^{2}\right), \quad i=n .
\end{array}\right.
$$

We then have for $1 \leq j \leq n$

$$
\left.\frac{\partial X_{i}}{\partial y_{j}}\right|_{y^{\prime}=0}=\left\{\begin{array}{l}
\left(1-2 s k_{i}(t)\right) \delta_{i j}, \quad i \neq n, \\
\frac{\partial \varphi}{\partial y_{j}}(t, 0)=0, \quad i=n .
\end{array}\right.
$$

In a small neighbourhood of $z^{*}(t)$, which is denoted by $V(t), \Gamma$ is represented as $x_{n}=\psi\left(x^{\prime}\right)$, and the mapping

$$
S(t) \cap U(t) \ni y=\left(y^{\prime}, \varphi\left(t, y^{\prime}\right)\right) \rightarrow X=\left(X^{\prime}, \psi\left(X^{\prime}\right)\right) \in \Gamma \cap V(t)
$$


is a diffeomorphism, which maps 0 to 0 (recall that $y^{*}(t)=(0, a(t))$ is mapped to $\left.z^{*}(t)=(0, a(t)+r(t))\right)$. For $y \in S(t) \cap U(t)$, let $s\left(t, y^{\prime}\right)<0$ be such that

$$
2 s\left(t, y^{\prime}\right) p\left(t, y^{\prime}\right)+y \in \Gamma \cap V(t) .
$$

Lemma 7.1. Let $x\left(t, y^{\prime}\right)=2 s\left(t, y^{\prime}\right) p\left(t, y^{\prime}\right)+\left(y^{\prime}, \varphi\left(t, y^{\prime}\right)\right)$. Then for $1 \leq i, j \leq n-1$,

$$
\begin{gathered}
\left.\frac{\partial x_{i}\left(t, y^{\prime}\right)}{\partial y_{j}}\right|_{y^{\prime}=0}=\left(1+2 r(t) k_{i}(t)\right) \delta_{i j} . \\
\left.\frac{\partial}{\partial x_{i}}\left(\psi\left(x^{\prime}\right)+\Phi_{2}\left(t, x^{\prime}, \psi\left(x^{\prime}\right)\right)\right)\right|_{x^{\prime}=0}=0 . \\
\left.\frac{\partial^{2}}{\partial x_{i} \partial x_{j}}\left(\psi\left(x^{\prime}\right)+\Phi_{2}\left(t, x^{\prime}, \psi\left(x^{\prime}\right)\right)\right)\right|_{x^{\prime}=0}=-\frac{2 k_{i}(t)}{1+2 r(t) k_{j}(t)} \delta_{i j} .
\end{gathered}
$$

Proof. Since $2 s(t, 0)=-\left|z^{*}(t)-y^{*}(t)\right|=-r(t)$ by (4.14), (7.1) follows from a direct computation. Using $\nabla_{x} \Phi_{2}(t, x)=p\left(t, y^{\prime}(x)\right)$, we have

$$
\frac{\partial}{\partial x_{i}} \Phi_{2}\left(t, x^{\prime}, \psi\left(x^{\prime}\right)\right)=p_{i}\left(t, y^{\prime}\left(x^{\prime}, \psi\left(x^{\prime}\right)\right)\right)+p_{n}\left(t, y^{\prime}\left(x^{\prime}, \psi\left(x^{\prime}\right)\right)\right) \frac{\partial \psi\left(x^{\prime}\right)}{\partial x_{i}} .
$$

Letting $x^{\prime}=0$ in the equation

$$
\begin{aligned}
& \frac{\partial}{\partial x_{i}}\left(\psi\left(x^{\prime}\right)+\Phi_{2}\left(t, x^{\prime}, \psi\left(x^{\prime}\right)\right)\right) \\
& =\left(1+p_{n}\left(t, y^{\prime}\left(x^{\prime}, \psi\left(x^{\prime}\right)\right)\right)\right) \frac{\partial \psi\left(x^{\prime}\right)}{\partial x_{i}}+p_{i}\left(t, y^{\prime}\left(x^{\prime}, \psi\left(x^{\prime}\right)\right)\right),
\end{aligned}
$$

we get (7.2). Taking the 2 nd order derivative, we have

$$
\frac{\partial^{2}}{\partial x_{i} \partial x_{j}}\left(\psi+\Phi_{2}\right)=\left(\frac{\partial}{\partial x_{j}} p_{n}\left(t, y^{\prime}\right)\right) \frac{\partial \psi}{\partial x_{i}}+\left(1+p_{n}\right) \frac{\partial^{2} \psi}{\partial x_{i} \partial x_{j}}+\frac{\partial}{\partial x_{j}} p_{i}\left(t, y^{\prime}\right) .
$$

Using (7.1), we have

$$
\frac{\partial}{\partial x_{j}} p_{i}\left(t,\left.y^{\prime}\left(x^{\prime}, \psi\left(x^{\prime}\right)\right)\right|_{x^{\prime}=0}=-\frac{2 k_{i}(t)}{1+2 r(t) k_{j}(t)} \delta_{i j} .\right.
$$

Since $p_{n}\left(t, y^{\prime}\right)=-1+O\left(\left|y^{\prime}\right|^{2}\right)$, we have

$$
\left.\frac{\partial}{\partial x_{j}} p_{n}\left(t, y^{\prime}\left(x^{\prime}, \psi\left(x^{\prime}\right)\right)\right)\right|_{x^{\prime}=0}=0 .
$$

We have thus proven (7.3).

In view of Theorem 6.4, in order to prove Theorem 1.1, we have only to compute

$$
B_{\Gamma}(t, \lambda):=\int_{\Gamma} h_{\mathrm{bw}}(t, x ; \lambda) \partial_{\nu} \sum_{j=1,2}\left(a_{j}(t, x ; \lambda) e^{\lambda^{2} t+\lambda \Phi_{j}(t, x)}\right) d \Gamma .
$$

Lemma 7.2. We have for $t>0$

$$
B_{\Gamma}(t, \lambda)=2 \lambda^{(3-n) / 2} \frac{1-k}{1+k}\left(\prod_{i=1}^{n-1} \frac{\pi}{k_{i}(t)}\right)^{1 / 2} e^{2 \lambda a(t)-r(t) \dot{a}(t)}\left(1+O\left(\frac{1}{\lambda}\right)\right) .
$$


Proof. Set

$$
B_{j}(t, \lambda)=\int_{\Gamma} h_{\mathrm{bw}}(t, x ; \lambda) \partial_{\nu}\left(a_{j}(t, x ; \lambda) e^{\lambda^{2} t+\lambda \Phi_{j}(t, x)}\right) d \Gamma, \quad j=2,3 .
$$

Letting $p=\left(p_{1}, \cdots, p_{n}\right)$ be as in (4.16), we have

$$
h_{\mathrm{bW}} \partial_{\nu}\left(a_{2} e^{\lambda^{2} t+\lambda \Phi_{2}}\right)=e^{\lambda\left(x_{n}+\Phi_{2}\right)}\left(\lambda a_{2} \nu \cdot p+\nu \cdot \nabla a_{2}\right)=: e^{\lambda\left(x_{n}+\Phi_{2}\right)} \lambda A_{\lambda}(t, x) .
$$

The integral (7.6) is performed in a small neighborhood of $z^{*}(t)$, and Lemma 7.1 shows that $x^{\prime}=0$ is a unique non-degenerate critical point of $\Phi\left(x^{\prime}\right)=x_{n}+\Phi_{2}(t, x)$, $x_{n}=\psi\left(x^{\prime}\right)$. Moreover we have

$$
\Phi(0)=2 a(t)
$$

and, thanks to (7.3),

$$
\left|\operatorname{det} \Phi^{\prime \prime}(0)\right|=\prod_{i=1}^{n-1} \frac{2 k_{i}(t)}{1+2 r(t) k_{i}(t)} .
$$

By Laplace's method, we have

$$
\begin{aligned}
B_{2}(t, \lambda) & \simeq\left(\frac{2 \pi}{\lambda}\right)^{(n-1) / 2} \frac{\left(1+\left|\nabla^{\prime} \psi\right|^{2}\right)^{1 / 2}}{\left|\operatorname{det} \Phi^{\prime \prime}(0)\right|^{1 / 2}} \lambda A_{\lambda}(t, 0) e^{2 \lambda a(t)} \\
& \simeq \lambda^{(3-n) / 2}(2 \pi)^{(n-1) / 2} \frac{\left(1+\left|\nabla^{\prime} \psi\right|^{2}\right)^{1 / 2} \nu \cdot p}{\left|\operatorname{det} \Phi^{\prime \prime}(0)\right|^{1 / 2}} a_{20}\left(t, z^{*}(t)\right) e^{2 \lambda a(t)} .
\end{aligned}
$$

Since $\left.\nu \cdot p\left(1+\left|\nabla^{\prime} \psi\right|^{2}\right)^{1 / 2}\right|_{x=z^{*}(t)}=1$, in view of Lemma $5.1(3 \mathrm{~b})$ and (7.7), we have proved

$$
B_{2}(t, \lambda)=\lambda^{(3-n) / 2} \frac{1-k}{1+k}\left(\prod_{i=1}^{n-1} \frac{\pi}{k_{i}(t)}\right)^{1 / 2} e^{2 \lambda a(t)-r(t) \dot{a}(t)}\left(1+O\left(\frac{1}{\lambda}\right)\right) .
$$

Consider $B_{3}(t, \lambda)$. Let $\bar{p}$ be as in the proof of Lemma 4.2. Thanks to (4.20) we have $\nu \bar{p}=\partial_{\nu} \Phi_{3}=-\partial_{\nu} \Phi_{2}$ on $\Gamma_{\epsilon}$ and, thanks to (5.14), $a_{3}=-a_{2}$ on $\Gamma_{\epsilon}$. Hence $B_{3}(t, \lambda)=B_{2}(t, \lambda)+O\left(\lambda^{(1-n) / 2}\right) e^{2 \lambda a(t)}$. Lemma 7.2 is proved.

By virtue of Lemma 7.2, we have

$$
\int_{T_{0}}^{T} e^{\lambda \mu t} B_{\Gamma}(t, \lambda) d t \sim \lambda^{(3-n) / 2} \frac{1-k}{1+k} \int_{0}^{T} e^{\lambda(\mu t+2 a(t))-r(t) \dot{a}(t)}\left(\prod_{i=1}^{n-1} \frac{\pi}{k_{i}(t)}\right)^{1 / 2} d t .
$$

7.2. Proof of Corollary 1.2. From Theorem 1.1, we have

$$
\frac{1}{\lambda} \log I_{\Gamma}\left(T_{0}, T ; \lambda, \mu\right) \rightarrow \mu T+2 a(T) .
$$

The right-hand side determines $a(t)$ for $0<t \leq T$, if $\left[T_{0}, T\right]$ is replaced by any small interval $\left[t_{0}, t\right]$ in $(0, T]$. Let us note that $a(t)$, etc. defined above depend also on $\mathbf{e}^{*}$, i.e. $a(t)=a\left(t, \mathbf{e}^{*}\right)$, etc. 
For $\mathbf{e} \in \mathbb{S}^{n-1}$ sufficiently close to $\mathbf{e}^{*}$, one can also recover $a(t, \mathbf{e})$. Let $\Pi(\mathbf{e})$ be the plane defined by $x \cdot \mathbf{e}=a(t, \mathbf{e})$. Then by taking a small neighborhood $U$ in $\mathbb{S}^{n-1}$ of $\mathbf{e}^{*}$, we have a family of planes $\{\Pi(\mathbf{e}) ; \mathbf{e} \in U\}$. The part of $\partial D(t)$ sufficiently close to $y^{*}\left(t, \mathbf{e}^{*}\right)$ can be reconstructed as the envelop of $\{\Pi(\mathbf{e}) ; \mathbf{e} \in U\}$. Then we can reconstuct $S(t, \mathbf{e})$ and $K(t, \mathbf{e})$. Finally using (1.12) again, we can recover the conductivity $k^{2}$.

7.3. Proof of Theorem 1.4. Let $\hat{\chi} \in C^{\infty}(\mathbb{R})$ be such that $\hat{\chi}(t)=1\left(t>T-\epsilon^{3} / 2\right)$, $\hat{\chi}(t)=0\left(t<T-\epsilon^{3}\right)$. If $a_{2}<a(T)$, we replace the ansatz $v_{a p r}(t, x)$ by $\hat{v}_{a p r}(t, x ; \lambda)=$ $\hat{\chi}(t) v_{a p r}(t, x ; \lambda)$ and set

$$
\begin{gathered}
\hat{I}_{\Gamma}\left(T_{0}, T ; \lambda, \mu\right):=\int_{T_{0}}^{T} e^{\lambda \mu t}\left(\int_{\Gamma} h_{\mathrm{bw}}(t, x ; \lambda) \partial_{\nu} \hat{v}_{a p r}(t, x ; \lambda) d \Gamma\right) d t \\
\delta \hat{I}_{\Gamma}\left(T_{0}, T ; \lambda, \mu\right):=\int_{T_{0}}^{T} e^{\lambda \mu t}\left(\int_{\Gamma} h_{\mathrm{bW}}(t, x ; \lambda) \partial_{\nu}\left(v_{a p r}(t, x ; \lambda)-\hat{v}_{a p r}(t, x ; \lambda)\right) d \Gamma\right) d t .
\end{gathered}
$$

This is estimated as

$$
\begin{aligned}
\left|\delta \hat{I}_{\Gamma}\left(T_{0}, T ; \lambda, \mu\right)\right| & \leq \int_{T_{0}}^{T-\epsilon^{3} / 2} e^{\lambda \mu t}\left|\int_{\Gamma} h_{\mathrm{bw}}(t, x ; \lambda) \partial_{\nu} v_{a p r}(t, x ; \lambda) d \Gamma\right| d t \\
& \leq C \lambda^{(3-n) / 2} \int_{T_{0}}^{T-\epsilon^{3} / 2} e^{\lambda \mu t+2 \lambda a(t)} d t \\
& \leq C \lambda^{(1-n) / 2} e^{\lambda \mu\left(T-\epsilon^{3} / 2\right)+2 \lambda a\left(T-\epsilon^{3} / 2\right)} .
\end{aligned}
$$

Since $\mu>\mu_{0}>2 \sup \{|\dot{a}(t)|, 0<t<T\}$, we then obtain

$$
\left|\delta \hat{I}_{\Gamma}\left(T_{0}, T ; \lambda, \mu\right)\right| \leq C \lambda^{-\alpha} e^{\lambda \mu T+2 \lambda a(T)}, \quad \forall \alpha \in \mathbb{R} .
$$

Thus $\widetilde{I}_{\Gamma}\left(T_{0}, T, \lambda, \mu\right)$ has the same behaviour as $\hat{I}_{\Gamma}\left(T_{0}, T, \lambda, \mu\right)$ when $\lambda$ goes to infinity. This shows that the ansatzs can be localized near $t=T$.

Consider the case $a_{2}<a(T)$, in which $\left\{x_{n}>a_{2}\right\} \subset\left\{x_{n}>a(T)-C \epsilon^{3}\right\}$ for $\epsilon$ sufficiently small. Then $\hat{v}_{a p r}$ vanishes in $\left\{(t, x) ; x_{n}-a(t) \leq-\epsilon^{3}\right.$, and $\left.t \leq T-\epsilon^{3}\right\}$, and so it vanishes in $\left\{(t, x) ; x_{n}-a(t) \leq-C \epsilon^{3}\right\}$ with $C=1+\mu_{0} / 2$. We then have

$$
\hat{I}_{\Gamma}\left(T_{0}, T ; \lambda, \mu\right)=\int_{T_{0}}^{T} e^{\lambda \mu t}\left(\int_{\Gamma \cap\left\{x_{n}>a(T)-C \epsilon^{3}\right\}} h_{\mathrm{bW}}(t, x ; \lambda) \partial_{\nu} \hat{v}_{a p r}(t, x ; \lambda) d \Gamma\right) d t .
$$

Thanks to (7.9), (7.10), we obtain (1).

Consider the case $a_{1}>a(T)$. We then simply replace the ansatz by $v=$ $\hat{\chi}(t) \widetilde{h_{\mathrm{fw}}}(t, x ; \lambda)$ in $(1.13)$, and have

$$
\partial_{t} v-\nabla \cdot(\gamma \nabla v)=\partial_{t} v-\Delta v=O\left(\lambda e^{\lambda^{2} T+\lambda a_{2}}\right) .
$$

The energy estimate shows that

$$
\left|\widetilde{I}_{\Gamma_{1}}\left(T_{0}, T ; \lambda, \mu\right)-\int_{T_{0}}^{T} e^{\lambda \mu t}\left(\int_{\Gamma} \widetilde{h_{\mathrm{bW}}}(t, x ; \lambda) \partial_{\nu} v(t, x ; \lambda) d \Gamma\right) d t\right| \leq C \lambda^{k} e^{\lambda \mu T+2 \lambda a_{2}},
$$


for some $k \in \mathbb{R}$. Hence

$$
\left|\widetilde{I}_{\Gamma_{1}}\left(T_{0}, T, \lambda, \mu\right)\right|=O\left(\lambda^{k}\right) e^{\lambda \mu T+2 \lambda a_{2}}
$$

Since $a_{2}>a_{1}>a(T)$ is arbitrary, we can replace $a_{2}$ in the above estimate by any $a_{3} \in\left(a_{1}, a_{2}\right)$, and so we obtain (2). We have finished the proof.

\section{REFERENCES}

[1] H. Bellout, Stability result for the inverse transmissitivity problem for the heat equation, J. Math. Anal. Appl. 168 (1992), 13-27.

[2] V. Bacchelli, M. Di Cristo, E. Sincich, S. Vessella, A paprbolic inverse problem with mixed boundary data. Stability estimates for the unknown boundary and impedance, preprin (2011).

[3] M. Di Cristo and S. Vessella, Stable determination of the discontinuous conductivity coefficient of a parabolic equation, arXiv:0904 (2009).

[4] Y. Daido, H. Kang and G. Nakamura, A probe method for the inverse boundary value problem of non-stationary heat equations, Inverse Problems 23 (2007), 1787-1800.

[5] Dashti Ardakani, Mohsen and Khodadad, Mahmud Identification of thermal conductivity and the shape of an inclusion using the boundary elements method and the particle swarm optimization algorithm, Inverse Problems in Science and Engineering, 17 (2009), pp. 855-870,

[6] A. Elayyan and V. Isakov, On uniqueness of the recovery of the discontinuous conductivity coefficient of a parabolic equation, SIAM. J. Math. Anal. 28 (1997), 49-59.

[7] P. Gaitan, H. Isozaki, O. Poisson, S. Siltanen and J. Tamminen, Inverse problems for timedependent singular heat conductivities - One dimensional case, preprint (2012).

[8] P. Gaitan, H. Isozaki, O. Poisson, S. Siltanen and J. Tamminen, Probing for inclusions in heat conductive bodies, Inverse Problems and Imaging 6 (2012), pp. 423-446.

[9] L. Hörmander, The Analysis of Linear Partial Differential Operators III, Springer-Verlag, Berlin-Heidelberg-New York-Tokyo (1985).

[10] T. Ide, H. Isozaki, S. Nakata, S. Siltanen, Local detection of three-dimensional inclusions in electrical impedance tomography, Inverse Porblems 26, (2010), 035001.

[11] T. Ide, H. Isozaki, S. Nakata, S. Siltanen and G. Uhlmann, Probing for electrical inclusions with complex spherical waves, CPAM Vol LX (2007), 1415-1442.

[12] M.Ikehata, Extracting discontinuity in a heat conductive body. One-space dimensional case, Applicable Analysis 86 (2007), 963-1005.

[13] M. Ikehata and M. Kawashita, The enclosure method for the heat equation, Inverse Problems 25 (2009), 075005.1

[14] H. Kawakami and M. Tsuchiya, Uniqueness and shape identification of a time-varying domain and related parabolic equation on non-cylidrical domains, Inverse Problems 26 (2010), 125007.

[15] J. L. Lions, Équations differéntielles opérationnelles et problèmes aux limites, SpringerVerlag, Berlin, Göttingen, Heidelberg (1961).

[16] Liu, Ji-Chuan and Wei, Ting, Moving boundary identification for a two-dimensional inverse heat conduction problem, Inverse Problems in Science and Engineering 19 (2011), pp. 11391154

[17] Liu, Ji-Chuan and Wei, Ting, The method of lines to reconstruct a moving boundary for a one-dimensional heat equation in a multilayer domain, Journal of Engineering Mathematics 71 (2011), pp. 157-170

[18] H. Tanabe, Equations of Evolution, Monographs and Studies in Mathematics, 6, Pitman, Boston, Mass.-London (1979).1

[19] T. Wei and Y.S.Li, An inverse boundary problem for one-dimensional heat equation with a multilayer domain Engineering Analysis with Boundary Elements 33 (2009) 225-232

[20] S. Vessella, Quantitative estimates of unique continuation for parabolic equations, determination of unknown time-varying boundaries and optimal stability extimates, Inverse Problems 28 (2008), 1-81. 
Aix Marseille Université de Provence, IUt, Aix-en-Provence, France

E-mail address: patricia.gaitan@univmed.fr

Institute of Mathematics, University of Tsukuba, Tsukuba, 305-8571, Japan

E-mail address: isozakih@math.tsukuba.ac.jp

Aix Marseille Université de Provence, France

E-mail address: poisson@cmi.univ-mrs.fr

Department of Mathematics and Statistics, University of Helsinki, Finland

E-mail address: samuli.siltanen@helsinki.fi

Department of Mathematics and Statistics, University of Helsinki, Finland

E-mail address: janne.p.tamminen@helsinki.fi 